\title{
A Política Fiscal Expansionista que contrai!
}

\section{The Expansionary Fiscal Policy that shrinks!}

\author{
Celso José Costa Júnior* \\ Amanda Miranda Fantinatti** \\ Vladimir Kuhl Teles***
}

Resumo: No presente trabalho, analisamos a desoneração do imposto sobre o consumo de bens duráveis com o objetivo de fornecer contribuições em duas direções: a) estimar e calibrar um modelo dynamic stochastic general equilibrium (DSGE) de pequena escala que incorpore esse aspecto; e b) analisar o impacto específico dessa política nas principais variáveis macroeconômicas à luz do modelo. Os resultados sugerem que a redução da alíquota do imposto sobre produtos industrializados (IPI) sobre bens duráveis não foi a política mais adequada para estimular a atividade econômica, pois contrai em vez de expandir o produto total. Melhor seria uma redução da alíquota do imposto indireto para toda a economia.

Palavras-chave: Política fiscal. Políticas anticíclicas. Desonerações tributárias. Bens duráveis. Modelos DSGE.

\begin{abstract}
In this paper, we analyze the tax exemption on consumption of durable goods aiming to contribute in two directions: a) estimate and calibrate a small scale DSGE model that incorporates this aspect; and b) evaluate the impact of this specific policy on the main macroeconomic variables through the lens of the model. The results suggest the impact of reducing the IPI rate on durable goods was not the most appropriate policy to stimulate economic activity, since it contracted instead of expanding the total product. Better would have being a reduction of the indirect tax rate for the whole economy.
\end{abstract}

Keywords: Fiscal policy. Countercyclical policy. Tax exemptions. Durable goods. DSGE models.

JEL Classification: E62; E30; C54.

1 Introdução

A eclosão da crise financeira internacional de 2008 colocou o uso da política fiscal novamente no radar dos formuladores de políticas públicas. Uma das principais razões para o estímulo fiscal é impulsionar o consumo quando a demanda agregada é percebida como fraca.

* $\quad$ Pós-doutorado em Economia pela Escola de Economia de São Paulo (EESP) da Fundação Getúlio Vargas (FGV). Doutor em Desenvolvimento Econômico pela Universidade Federal do Paraná (UFPR). Professor do Departamento de Economia da Universidade Estadual de Ponta Grossa (UEPG).E-mail: cjcjunior@uepg.br

** Mestre em Economia pela Escola de Economia de São Paulo (EESP) da Fundação Getúlio Vargas (FGV).E-mail: miranda.a.amanda@gmail.com

*** Doutor em Economia pela Universidade de Brasília (UnB). Professor da Escola de Economia de São Paulo (EESP) da Fundação Getúlio Vargas (FGV). E-mail: vladimir.teles@fgv.br 
Em economias avançadas, a política fiscal foi usada, muitas vezes, com o objetivo de aumentar a poupança nacional e obter o reequilíbrio externo, tendo como preocupação a manutenção da sustentabilidade fiscal e a dívida pública em níveis adequados no longo prazo. Para contrabalancear flutuações do ciclo econômico, a política monetária era o instrumento central.

Após a crise financeira global de 2008 iniciada nos Estados Unidos ter se espalhado rapidamente pelo mundo, diversos bancos centrais adotaram políticas monetárias expansionistas em resposta à forte recessão que se observava. Tendo atingido o limite inferior das taxas de juros nominais (zero lower bound) e, nesse caso, a política monetária se revelando ineficaz para reanimar a atividade econômica, grande parte dos países atingidos pelo pânico financeiro recorreu a diversos estímulos fiscais e a políticas monetárias não convencionais a fim de evitar uma contração da atividade ainda mais pronunciada.

No presente trabalho, analisamos uma forma específica de intervenção na economia, as desonerações do imposto sobre consumo de bens duráveis, isto é, a redução na alíquota do imposto sobre produtos industrializados (IPI) para incentivar a compra desse tipo de bem. O objetivo é fornecer contribuições em duas direções: a) estimar e calibrar um modelo dynamic stochastic general equilibrium (DSGE) de pequena escala que incorpore esse aspecto; e b) analisar o impacto específico dessa política nas principais variáveis macroeconômicas à luz do modelo.

A escolha desse tema se dá pelo fato da desoneração do IPI sobre o setor automotivo ser um dos primeiros estímulos fiscais anunciado após a crise. Vale destacar, também, a importância do setor para a produção industrial, em particular, e para a economia, em geral. Ademais, essa medida exemplifica a escolha do governo em direcionar estímulos a setores específicos da economia. França, Itália e Espanha (ADDA; COOPER, 2000) e Estados Unidos (MIAN; SUFI, 2012) também tiveram programas similares e com resultados semelhantes aos apresentados no Brasil. Adicionalmente, o debate sobre o efeito de estímulos fiscais é mais relevante em períodos de recessão, e essa intervenção entrou em vigor nesse período.

É interessante notar que a adoção de políticas fiscais expansionistas foi incentivada, inclusive, por organizações internacionais, tendo sido umas das recomendações de política econômica do comunicado do G-20, em 15 de novembro de 2008:

Against this background of deteriorating economic conditions worldwide, we agreed that a broader policy response is needed, based on closer macroeconomic cooperation, to restore growth, avoid negative spillovers and support emerging market economies and developing countries. As immediate steps to achieve these objectives, as well as to address longer-term challenges, we will: [...] Use fiscal measures to stimulate domestic demand to rapid effect, as appropriate, while maintaining a policy framework 
conducive to fiscal sustainability (UNIVERSITY OF TORONTO, 2008, não paginado).

As medidas adotadas foram heterogêneas entre os países em relação aos tipos de estímulo, tamanho e extensão dos programas. Dentre as economias avançadas, Austrália, Canadá, Alemanha, Japão, Coreia do Sul e Estados Unidos lançaram pacotes de estímulo fiscal que excederam 3\% do PIB (das economias do G-20) de 2008 em políticas discricionárias ao longo de 2009 e 2010 (INTERNATIONAL MONETARY FUND, 2010). Segundo estimativa de Saha e Weizsacker (2009), o tamanho dos pacotes de estímulos adotados para combater a crise, somente em 2009 , chegou a $€ 129,6$ bilhões na zona do euro (ou 1,0\% PIB) e a $€ 186,0$ bilhões (ou 1,7\% PIB) nos Estados Unidos.

No Brasil, a exemplo do ocorrido no mundo, a crise financeira que se instaurou no terceiro trimestre de 2008 ensejou diversas respostas de política econômica. Medidas anticíclicas de desoneração tributária e aumento de gastos públicos com o intuito de amenizar os efeitos da crise foram adotadas, totalizando cerca de $\mathrm{R} \$ 12,5$ bilhões ( $0,4 \%$ PIB) e $\mathrm{R} \$ 30,9$ bilhões (1,0\% PIB), respectivamente, em 2009 (PIRES, 2009). Dentre as medidas de desoneração tributária, o governo brasileiro, a exemplo do ocorrido na França, Alemanha, Inglaterra e Estados Unidos, adotou, em dezembro de 2008, medidas para incentivar a compra de automóveis novos, visando à redução dos estoques desse setor, que haviam aumentado expressivamente em virtude da queda da demanda. Diferentemente dos países citados, no Brasil o incentivo se deu por meio da desoneração temporária da alíquota de impostos indiretos (por exemplo, do IPI) sobre automóveis, inicialmente, até março de 2009, conforme mostra a Tabela 1.

Tabela 1 - Mudanças na alíquota de IPI para automóveis

\begin{tabular}{l|c|c|c|c|c|c|c|c|c}
\hline & $\begin{array}{c}\text { Alíquota } \\
\text { que vi- } \\
\text { geripo de } \\
\text { geículo } \\
\text { antes da } \\
\text { crise }\end{array}$ & $\begin{array}{c}\text { Dez. } \\
\mathbf{2 0 0 8}\end{array}$ & $\begin{array}{c}\text { Out. } \\
\mathbf{2 0 0 9}\end{array}$ & $\begin{array}{c}\text { Nov. } \\
\mathbf{2 0 0 9}\end{array}$ & $\begin{array}{c}\text { Abr. } \\
\mathbf{2 0 1 0}\end{array}$ & $\begin{array}{c}\text { Maio } \\
\mathbf{2 0 1 2}\end{array}$ & $\begin{array}{c}\text { Jan. } \\
\mathbf{2 0 1 3}\end{array}$ & $\begin{array}{c}\text { Jan. } \\
\mathbf{2 0 1 4}\end{array}$ & $\begin{array}{c}\text { Jan. } \\
\mathbf{2 0 1 5}\end{array}$ \\
\hline $\begin{array}{l}1.0 \text { a álcool } \\
1.0 \text { a } 2.0 \text { a }\end{array}$ & $7.0 \%$ & $0.0 \%$ & $1.5 \%$ & $3.0 \%$ & $7.0 \%$ & $0.0 \%$ & $2.0 \%$ & $3.0 \%$ & $7.0 \%$ \\
$\begin{array}{l}\text { álcool } \\
1.0 \text { a } 2.0 \text { a } \\
\text { gasolina }\end{array}$ & $11.0 \%$ & $5.5 \%$ & $6.5 \%$ & $7.5 \%$ & $11.0 \%$ & $5.5 \%$ & $7.0 \%$ & $9.0 \%$ & $11.0 \%$ \\
\hline
\end{tabular}

Fonte: Elaboração própria a partir de RFB (2015).

Ao final de março de 2009, o Ministério da Fazenda (CONJUR) anunciou a manutenção da desoneração para o setor automotivo, assim como a ampliação para outros setores. De fato, esse mecanismo, inicialmente adotado por um tri- 
mestre, foi prorrogado por diversas vezes. A medida teve um efeito positivo em estimular novas vendas - principalmente após dezembro de 2008 e maio de 2012, época na qual a alíquota dos veículos mais básicos foi zerada. No entanto, as vendas de veículos novos cresciam a taxas decrescentes, devido a certa antecipação do consumo. O governo, assim, era levado a prorrogar o incentivo com receio de uma queda pronunciada nas vendas na ausência da medida.

Há enorme incerteza na literatura a respeito dos efeitos da política fiscal expansionista sobre o nível de atividade. Perotti et al. (2007) afirmam que muitos economistas concordariam que uma elevação da taxa de juros causaria uma redução tanto na taxa de inflação da economia quanto em sua taxa de crescimento, e, além disso, a maior parte da evidência empírica é consistente com essa afirmação. No entanto, muitos economistas discordam dos possíveis impactos de um choque nos gastos do governo. Essa incerteza decorre das diversas hipóteses teóricas sobre a relação entre política fiscal e atividade econômica, assim como da dificuldade em se estimar tal relação (CAVALCANTI, 2009).

Cogan et al. (2010), baseados no modelo DSGE desenvolvido por Smets e Wouters (2003), concluem que os multiplicadores de um aumento permanente dos gastos do governo federal são muito menores nos modelos novo-keynesianos do que nos modelos keynesianos mais antigos. O impacto dessa política é muito pequeno no primeiro ano e, como o gasto do governo se reduz nos anos subsequentes da simulação, os multiplicadores se tornam negativos. De maneira similar, Forni et al. (2009) encontram evidências de efeitos apenas moderados da política fiscal na economia. De acordo com esses autores, políticas de aumento de gastos públicos com bens e serviços e remuneração de funcionários públicos têm efeito pequeno e de curta duração no consumo privado, enquanto inovações em transferências às famílias mostram um efeito mais pronunciado e duradouro. No entanto, os resultados sugerem que uma redução nos impostos incidentes no rendimento do trabalho e no consumo tem efeito considerável no consumo e no produto agregado, enquanto uma redução nos impostos incidentes na renda do capital favorece tanto o investimento quanto o produto no médio prazo. Assim, com exceção de transferências para as famílias e das taxas de imposto sobre o rendimento do trabalho, a maioria das variáveis de política fiscal pouco contribui para a variabilidade cíclica das principais variáveis macroeconômicas.

Os efeitos da política fiscal também têm recebido atenção na literatura nacional. O resultado encontrado por Peres (2006), apoiado na especificação de Blanchard e Perotti (2002) para o período entre 1994 e 2005 para a economia nacional, mostra que a resposta do produto a choques fiscais é pequena e tem características previstas pela teoria keynesiana, isto é, é positiva dado um choque nos gastos públicos (federal) e negativa diante de um choque na carga tributária líquida. Mendonça, Medrano e Sachsida (2009) utilizam a abordagem de Moun- 
tford e Uhlig (2005) na tentativa de diferenciar os efeitos dos choques de política fiscal dos demais choques na economia. Os resultados sugerem que um aumento inesperado dos gastos do governo levaria ao aumento do consumo privado e a uma redução do PIB com uma alta probabilidade (indicando efeito crowding out entre investimento público e privado). No entanto, um choque positivo na carga tributária líquida pode levar, a médio prazo, a uma resposta positiva do PIB.

Carvalho e Valli (2011) utilizam um modelo DSGE que incorpora uma meta de superávit primário, impostos distorcivos (sobre consumo, trabalho, capital e dividendos), programas sociais na forma de transferências públicas e investimento do governo. De forma a permitir que a política fiscal tenha efeito no consumo agregado, os autores introduzem agentes não ricardianos no modelo. Para levar em consideração que a variável fiscal principal no Brasil é o resultado primário (em \% do PIB), os autores utilizam uma regra de superávit primário que reage ao ciclo econômico e a desvios da dívida pública (em \% do PIB) em relação ao estado estacionário - similarmente à especificação do presente trabalho. Os autores concluem que a maioria dos efeitos de um choque expansionista do superávit primário (em \% do PIB), inclusive no produto, desaparece dentro de dois anos. No entanto, o aumento da dívida pública (em \% do PIB) é duradouro.

Cavalcanti e Vereda (2014), através de um modelo DSGE, quantificam e comparam diferentes tipos de gastos públicos (compras de bens e serviços, investimento público, transferências sociais e emprego público) sob diferentes regras fiscais. Dentre os resultados encontrados, destacam-se: a) nenhum tipo de gasto público gera impacto positivo significativo no PIB, no curto prazo, sob as regras de ajuste fiscal baseadas na redução de algum item de despesa; b) a maioria dos itens de gasto público caracteriza-se por multiplicadores pouco significativos ou negativos sob a política de equilíbrio orçamentário permanente, mas positivos sob a política de ajuste fiscal defasado e parcial.

Mian e Sufi (2010), ao analisar a política de incentivos à compra de veículos novos nos Estados Unidos em 2009, concluem que a medida não teve efeito de longo prazo na venda de veículos. Houve apenas uma antecipação das compras que seriam realizadas no futuro - e a um custo fiscal não desprezível. Vale ressaltar que os autores utilizam microdados e uma metodologia bastante distinta da aqui proposta. Os autores adicionam que seu resultado obtido é consistente com modelos que incorporam a hipótese da equivalência ricardiana, como o de Barro (1974, 1979). No entanto, os autores destacam, ainda, que a medida analisada é apenas uma forma de estímulo fiscal, então é plausível que haja outras formas de expansão fiscal de efeito mais duradouro.

Os resultados do modelo aqui proposto sugerem que o impacto da redução da alíquota do IPI sobre bens duráveis não foi a política mais adequada para estimular a atividade econômica. Com o impacto da desoneração desse imposto, 
o produto agregado recua para um valor abaixo do seu estado estacionário. Por outro lado, supondo que o choque de política fiscal tivesse sido uma redução da alíquota do imposto indireto para toda a economia ${ }^{1}$ ou para o setor de bens não duráveis e serviços (que detém uma participação maior na economia), o modelo aqui proposto sugere que essa política teria sido mais adequada para reanimar a atividade econômica.

Além desta introdução, este trabalho possui mais quatro seções: a seção 2 desenvolve o modelo econômico; a seção 3 exibe os procedimentos da estimação (dados, calibragem, valores prior e posterior dos parâmetros); a seção 4 discute os resultados; e a seção 5 apresenta as considerações finais.

\section{Modelo}

Nesta seção é apresentado o modelo utilizado para analisar políticas de subsídios governamentais, como a redução em impostos indiretos (IPI) após a eclosão da crise financeira mundial de 2008. O modelo aqui descrito segue muitas das características presentes nos trabalhos de Smets e Wouters (2003), Castro et al. (2011), Carvalho e Valli (2011), Cavalcanti e Vereda (2014), Teles, Costa Júnior e Rosa (2015) e Whelan (2003).

As famílias poupam através de empréstimos às instituições financeiras, que, por sua vez, repassam os empréstimos às firmas intermediárias que utilizam esses recursos como capital de giro para contratação e pagamento de salário de trabalhadores.

A produção da economia é concentrada nas firmas produtoras de bens intermediários para serem utilizados na montagem de bens de consumo, que podem ser de dois tipos: bens de consumo duráveis; e bens de consumo não duráveis e serviços. As firmas produtoras de bens intermediários contratam trabalhadores e capital e são sujeitas a choques de produtividade específicos no setor de bens duráveis e no setor de bens não duráveis e serviços. As firmas produtoras de bens finais apenas agregam a produção das firmas produtoras de bens intermediários, de forma que podem ser vistas como montadoras, e a sua função no modelo é permitir a incorporação de rigidez de preços de forma tratável. As firmas produtoras de bens de capital são responsáveis pela transformação de uma cesta de bens de investimento (I) em capital $(K)$.

Nesse modelo, o setor de bens duráveis apenas produz bens de consumo para as famílias, enquanto o setor de bens não duráveis e serviços produz bens de consumo das famílias e do governo e de investimento para as firmas produtoras de bens de capital.

1 A literatura sobre finanças públicas adverte que não usar a mesma alíquota para setores diferentes não é ótimo de Pareto (LEACH, 2004, p. 249) 
Uma característica importante dessa economia de dois setores é que os agentes não somente têm que escolher entre consumo e lazer no tempo (escolha intertemporal), mas também o tipo de bem (durável ou não durável e serviço) que será consumido no mesmo instante no tempo (escolha intratemporal). Dessa maneira, o preço relativo entre os dois tipos de bens terá um papel relevante na escolha dos agentes.

O governo divide-se em autoridade fiscal e monetária. O lado fiscal do governo realiza três funções: consome bens finais não duráveis e serviços, emite títulos e tributa as famílias através de impostos diretos e indiretos.

Segundo o IBGE, o conceito de consumo de governo se refere às despesas com bens e serviços oferecidos gratuitamente, total ou parcialmente, às famílias, que compõem a conta de uso da renda. Assim, uma hipótese fundamental do modelo é a de que o consumo do governo consiste apenas de bens não duráveis e serviços, que, apesar de forte, está em consonância com a descrição de consumo de governo do IBGE.

Por fim, do lado monetário, a taxa de juros é definida por meio de uma regra de Taylor.

\subsection{Familias}

As famílias recebem a renda do trabalho e de juros sobre depósitos bancários no sistema financeiro realizado no período anterior. Dessa maneira, as famílias decidem, no início do período, se usarão seus recursos para consumir (bens duráveis ou não duráveis e serviços) ou se farão depósitos nas instituições financeiras para receber juros no próximo período.

As famílias são caracterizadas por um contínuo de famílias idênticas, que vivem infinitamente, e cada família representativa i busca maximizar a função de utilidade esperada dada por

$$
\max _{C_{D, t}, C_{N D, t} L_{t}, N_{t+1}} E_{t} \sum_{t=0}^{\infty} \beta^{t} S_{t}^{P}\left(\frac{C_{t}^{1-\eta}}{1-\eta}-S_{t}^{L} \frac{L_{t}^{1+\omega}}{1+\omega}\right)
$$

com

$$
C_{t}=\left[\gamma_{D} D_{t+1}^{\frac{1}{\varphi_{D}}}+\left(1-\gamma_{D}\right) C_{N D, t}^{\frac{1}{\varphi_{D}}}\right]^{\varphi_{D}}
$$

e

$$
\mathrm{D}_{\mathrm{t}+1}=\mathrm{C}_{\mathrm{D}, \mathrm{t}}+\left(1-\delta_{\mathrm{D}}\right) \mathrm{D}_{\mathrm{t}}
$$

em que $\beta$ é o fator de desconto intertemporal, $C_{t}$ simboliza o consumo que depende do estoque de bens duráveis $\left(D_{t}\right)$ e do consumo de bens não duráveis 
e serviços $\left(C_{N D, t}\right), L_{t}$ representa o número de horas de trabalho ofertado, $\eta$ é o coeficiente de aversão relativa ao risco, $\omega$ é a desutilidade marginal do trabalho, $\gamma_{D}$ é a participação de bens duráveis no consumo das famílias, $\varphi_{D}$ é a elasticidade de substituição entre os bens de consumo duráveis e não duráveis e serviços, $\delta_{D}$ é a taxa de depreciação dos bens duráveis e $S_{t}^{P}$ e $S_{t}^{L}$ são variáveis estocásticas que incorporam choques na preferência intertemporal e na oferta de trabalho, respectivamente, sendo que seguem um processo autoregressivo:

$$
\log S_{t}^{P}=\left(1-\rho^{P}\right) \log S_{s s}^{P}+\rho^{P} \log S_{t-1}^{P}+\epsilon_{t}^{P}
$$

em que $\rho^{\mathrm{P}}$ é o componente autorregressivo e $\epsilon_{t}^{\mathrm{P}} \sim \mathrm{N}\left(0, \sigma^{\mathrm{P}}\right)$.

e

$$
\log \mathrm{S}_{t}^{\mathrm{L}}=\left(1-\rho^{\mathrm{L}}\right) \log \mathrm{S}_{s \mathrm{~s}}^{\mathrm{L}}+\rho^{\mathrm{L}} \log \mathrm{S}_{\mathrm{t}-1}^{\mathrm{L}}+\epsilon_{\mathrm{t}}^{\mathrm{L}}
$$

em que $\rho^{\mathrm{L}}$ é o componente autorregressivo e $\epsilon_{\mathrm{t}}^{\mathrm{L}} \sim \mathrm{N}\left(0, \sigma^{\mathrm{L}}\right)$.

As famílias estão sujeitas à restrição orçamentária:

$$
\mathrm{C}_{\mathrm{D}, \mathrm{t}} \mathrm{P}_{\mathrm{D}, \mathrm{t}}\left(1+\tau_{\mathrm{D}, \mathrm{t}}^{\mathrm{II}}\right)+\mathrm{C}_{\mathrm{ND}, \mathrm{t}} \mathrm{P}_{\mathrm{ND}, \mathrm{t}}\left(1+\tau_{\mathrm{ND}, \mathrm{t}}^{\mathrm{II}}\right)+\mathrm{N}_{\mathrm{t}+1}=\mathrm{W}_{\mathrm{t}} \mathrm{L}_{\mathrm{t}}+\mathrm{R}_{\mathrm{t}}^{\mathrm{n}} \mathrm{N}_{\mathrm{t}}-\mathrm{T}_{\mathrm{t}}
$$

em que $\mathrm{P}_{\mathrm{D}, \mathrm{t}}$ é o nível de preços no setor de bens duráveis, $\mathrm{P}_{\mathrm{ND}, \mathrm{t}}$ é o nível de preços no setor de bens não duráveis e serviços, $W_{t}$ é o nível dos salários, $N_{t}$ representa a poupança, isto é, os depósitos remunerados do período anterior, $\mathrm{R}_{\mathrm{t}}^{\mathrm{n}}$ é a taxa de juros nominal bruta paga pelas instituições financeiras às famílias, $T_{t}$ simboliza os impostos diretos (lump-sum) pagos pelas famílias ao governo, $\tau_{\mathrm{ND}, \mathrm{t}}^{\mathrm{II}}$ é a alíquota do imposto indireto do setor de bens duráveis pago pelas famílias ao governo e, por fim, $\tau_{\mathrm{ND}, \mathrm{I}}^{\mathrm{II}}$ é a alíquota do imposto indireto do setor de bens não duráveis e serviços pago pelas famílias ao governo.

A família, ao resolver seu problema de maximização, encontra as seguintes equações:

$$
\begin{gathered}
\left(\frac{\gamma_{D}}{1-\gamma_{D}}\right)\left(\frac{D_{t+1}}{C_{N D, t}}\right)^{\frac{1-\varphi_{D}}{\varphi_{D}}}=\frac{P_{D, t}\left(1+\tau_{D, t}^{I I}\right)}{P_{N D, t}\left(1+\tau_{N D, t}^{I I}\right)} \\
\left(\frac{1}{1-\gamma_{D}}\right) S_{t}^{L} L_{t}^{\omega} C_{t}^{\eta}\left(\frac{C_{t}}{C_{N D, t}}\right)^{\frac{1-\varphi_{D}}{\varphi_{D}}}=\frac{W_{t}}{P_{N D, t}\left(1+\tau_{N D, t}^{I I}\right)}
\end{gathered}
$$




$$
\left[\frac{S_{t}^{P} C_{t}^{-\eta}}{P_{N D, t}\left(1+\tau_{N D, t}^{I I}\right)}\right]\left(\frac{C_{N D, t}}{C_{t}}\right)^{\frac{1-\varphi_{D}}{\varphi_{D}}}=\beta E_{t}\left\{\left[\frac{S_{t+1}^{P} C_{t+1}^{-\eta}}{P_{N D, t+1}\left(1+\tau_{N D, t+1}^{I I}\right)}\right]\left(\frac{C_{N D, t+1}}{C_{t+1}}\right)^{\frac{1-\varphi_{D}}{\varphi_{D}}} R_{t+1}^{n}\right\}
$$

A equação 7 é a de demanda relativa entre bens duráveis e não duráveis, a equação 8 é a da oferta de trabalho das famílias e a equação 9 é de Euler para o consumo.

\subsection{Firmas}

As firmas produzem os bens e serviços que as famílias irão consumir. Como já mencionado, o setor produtivo é subdividido em três tipos de firmas: a) as produtoras de bens finais (montadoras); b) as produtoras de bens intermediários; e c) as produtoras de bens de capital.

\subsubsection{Firmas Produtoras de Bens Finais ( $V$ arejistas)}

De uma perspectiva agregada, a competição monopolística envolve, entre outras coisas, confrontar o fato de que consumidores compram uma grande variedade de bens, mas, por finalidade de modelagem, assume-se que compram apenas um bem específico (agregado). Esse bem é vendido pelas firmas em uma estrutura de competição perfeita.

Com o objetivo de produzir esse bem agregado, ${ }^{2}$ a firma varejista deve comprar uma grande quantidade de bens intermediários. Esses são os insumos usados nesse processo produtivo. Dessa forma, a firma montadora deve resolver o seguinte problema:

$$
Y_{x, t}=\left(\int_{0}^{1} Y_{x, j, t}^{\frac{\varphi-1}{\varphi}} d j\right)^{\frac{\varphi}{\varphi-1}}
$$

no qual $Y_{x, t}$ é o produto agregado, $Y_{x, j, t}$ é o produto intermediário da firma j, $\varphi>1$ é a elasticidade de substituição entre bens intermediários e $x=\{D, N D\}$ representa $o$ tipo de bem produzido, durável ou não durável e serviço.

As firmas maximizam o lucro sujeito à restrição de tecnologia (Equação (10)):

$$
\max _{Y_{x, j, t}} P_{x, t} Y_{x, t}-\int_{0}^{1} P_{x, j, t} Y_{x, j, t} d j
$$

2 Existem dois tipos de firmas produtoras de bens finais na economia: as produtoras de bens de consumo duráveis $\left(\mathrm{C}_{\mathrm{D}, \mathrm{t}}\right)$ e as produtoras de bens de consumo não duráveis e serviços $\left(\mathrm{C}_{\mathrm{ND}, \mathrm{t}}\right)$. 
e o resultado nos fornece a demanda pelo bem intermediário j, dada por:

$$
Y_{x, j, t}=Y_{x, t}\left(\frac{P_{x, t}}{P_{x, j, t}}\right)^{\varphi}
$$

\subsubsection{Firmas Produtoras de Bens Intermediários (Atacadistas)}

As firmas produtoras de bens intermediários combinam trabalho e capital para produzir bens de consumo duráveis e bens de consumo não duráveis e serviços diferenciados sob competição monopolística. A função de produção das firmas produtoras de bens intermediários é dada por:

$$
Y_{x, j, t}=A_{x, t}\left(U_{t} K_{x, j, t}\right)^{\alpha} L_{x, j, t}^{1-\alpha}
$$

na qual $A_{x, t}$ representa a produtividade total dos fatores, $U_{t}$ é a taxa de utilização do capital, $K_{x, j, t}$ é o estoque de capital, $L_{x, j, t}$ é o trabalho e $\alpha$ e $1-\alpha$ representam a participação do capital e do trabalho na produção, respectivamente, em cada um dos setores dessa economia, isto é, no setor de bens duráveis ou bens não duráveis e serviços.

A produtividade total dos fatores é sujeita a choques específicos em cada setor:

$$
\log \mathrm{A}_{\mathrm{D}, \mathrm{t}}=\left(1-\rho^{\mathrm{A}, \mathrm{D}}\right) \log \mathrm{A}_{\mathrm{D}, \mathrm{SS}}+\rho^{\mathrm{A}, \mathrm{D}} \log \mathrm{A}_{\mathrm{D}, \mathrm{t}-1}+\epsilon_{\mathrm{t}}^{\mathrm{A}, \mathrm{D}}
$$

em que $\rho_{A, D}$ é o componente autoregressivo e $\epsilon_{t}^{\mathrm{A}, \mathrm{D}} \sim \mathrm{N}\left(0, \sigma^{\mathrm{A}, \mathrm{D}}\right)$.

e

$$
\log \mathrm{A}_{\mathrm{ND}, \mathrm{t}}=\left(1-\rho^{\mathrm{A}, \mathrm{ND}}\right) \log \mathrm{A}_{\mathrm{ND}, \mathrm{ss}}+\rho^{\mathrm{A}, \mathrm{ND}} \log \mathrm{A}_{\mathrm{ND}, \mathrm{t}-1}+\epsilon_{\mathrm{t}}^{\mathrm{A}, \mathrm{ND}}
$$

em que $\rho_{A, N D}$ é o componente autoregressivo e $\epsilon_{t}^{\mathrm{A}, \mathrm{ND}} \sim \mathrm{N}\left(0, \sigma^{\mathrm{A}, \mathrm{ND}}\right)$.

Cada firma determina as escolhas dos insumos a serem utilizados, de forma a minimizar o custo sujeito à função de produção (equação 13):

$$
\min _{\mathrm{x}, \mathrm{j}, \mathrm{t}} \mathrm{K}_{\mathrm{x}, \mathrm{t}, \mathrm{t}} \mathrm{R}_{\mathrm{t}}^{\mathrm{f}} \mathrm{W}_{\mathrm{t}} \mathrm{L}_{\mathrm{x}, \mathrm{j}, \mathrm{t}}+\mathrm{R}_{\mathrm{t}} \mathrm{K}_{\mathrm{x}, \mathrm{j}, \mathrm{t}}
$$


na qual $R_{t}$ é o retorno do capital e $R_{-}{ }^{\wedge}{ }^{\wedge}$ é a remuneração do capital de giro utilizada para a contratação e pagamento de salário dos trabalhadores, isto é, a taxa de juros bruta paga pelas firmas intermediárias às instituições financeiras (IFs). É importante observar que o custo das empresas é afetado pela taxa de juros cobrada pelas IFs, e, assim, choques de liquidez afetam as decisões de alocação das firmas.

Desse problema, temos as condições de que as remunerações dos fatores de produção serão pagas de acordo com os respectivos retornos marginais:

$$
\begin{gathered}
L_{x, j, t}=(1-\alpha) C M_{x, t} \frac{Y_{x, j, t}}{R_{t}^{f} W_{t}} \\
U_{t} K_{x, j, t}=\alpha C M_{x, t} A_{x, t} \frac{Y_{x, j, t}}{R_{t}}
\end{gathered}
$$

O índice j é omitido ao assumirmos um equilíbrio simétrico.

Adicionalmente, $C M_{x, t}$ representa o custo marginal em cada um dos setores dessa economia e pode ser expresso pela equação:

$$
C M_{x, j, t}=\frac{1}{A_{x, t}}\left(\frac{R_{t}}{\alpha}\right)^{\alpha}\left(\frac{R_{t}^{f} W_{t}}{1-\alpha}\right)^{1-\alpha}
$$

Supõe-se que a firma intermediária decide o quanto produzir em cada período de acordo com uma regra de rigidez de preços de acordo com Calvo (1983). Essa regra afirma que, em cada período $t$, uma fração $(1-\theta)$, com $0<\theta<1$ das firmas são aleatoriamente selecionadas e podem escolher o preço do seu bem para o período $t$. O restante $\theta$ das firmas mantém o preço do período anterior.

Assim, o problema da firma capaz de reajustar o preço do seu bem é:

$$
\max _{P_{x, j, t}^{*}} E_{t} \sum_{i=0}^{\infty}(\beta \theta)^{i} Y_{x, t+1}\left(\frac{P_{x, t+1}}{P_{x, j, t}^{*}}\right)^{\varphi}\left(P_{x, j, t}^{*}-C M_{x, j, t}\right)
$$

A condição de primeira ordem é:

$$
P_{x, j, t}^{*}=\left(\frac{\varphi}{\varphi-1}\right) E_{t} \sum_{i=0}^{\infty}(\beta \theta)^{i} C M_{x, j, t}
$$

O nível de preços é dado por:

$$
P_{x, t}=\left[\theta P_{x, t-1}^{1-\varphi}+(1-\theta) P_{x, t}^{1-\varphi}\right]^{\frac{1}{1-\varphi}}
$$




\subsubsection{Firmas Produtoras de Bens de Capital}

A acumulação de capital é responsabilidade de uma única firma, que transforma uma cesta de bens de investimento ( $I$ ) em capital (K). Essa firma define a quantidade $I$ a ser transformada em capital maximizando o lucro auferido na cessão de capital para as firmas intermediárias, sujeito a fricções reais como custos de ajustamento do capital e utilização variável da capacidade instalada. Portanto, essa firma deve resolver o problema:

$$
\max _{U_{t}, K_{t+1}, I_{t}} E_{t} \sum_{t=0}^{\infty} \Xi_{0, t}\left\{R_{t} U_{t} K_{t}-P_{t} K_{t}\left[\psi_{1}\left(U_{t}-1\right)+\frac{\psi_{2}}{2}\left(U_{t}-1\right)^{2}\right]-P_{t} I_{t}\right\}
$$

sujeito à lei de movimento do capital:

$$
K_{t+1}=(1-\delta) K_{t}+I_{t}\left[1-\frac{\chi}{2}\left(\frac{I_{t}}{S_{t}^{I} I_{t-1}}-1\right)^{2}\right]
$$

em que $\Xi_{0, t}$ é o fator de desconto estocástico utilizado para trazer a valor presente os recebimentos futuros esperados, $U$ é a taxa de utilização do capital, I é o investimento, $\psi_{1}$ e $\psi_{2}>0$ são parâmetros de sensibilidade da utilização da capacidade instalada, é o parâmetro de sensibilidade dos investimentos e é a produtividade do investimento cuja lei de movimento é dada por:

$$
\log S_{t}^{I}=\left(1-\rho_{I}\right) \log S_{S S}^{I}+\rho_{I} \log S_{t-1}^{I}+\epsilon_{I, t}
$$

na qual $\rho_{\mathrm{I}}$ é o componente autorregressivo e $\epsilon_{\mathrm{I}, \mathrm{t}} \sim \mathrm{N}\left(0, \sigma^{\mathrm{I}, \mathrm{t}}\right)$.

Resolvendo-se o problema da firma produtora de bens de capital, chega-se às condições de primeira ordem:

$$
\begin{gathered}
\frac{R_{t}}{P_{t}}=\psi_{1}+\psi_{2}\left(U_{t}-1\right) \\
Q_{t}=E_{t} \Xi_{t, t+1}\left\{(1-\delta) Q_{t+1}+R_{t+1} U_{t+1}-P_{t+1}\left[\psi_{1}\left(U_{t+1}-1\right)+\frac{\psi_{2}}{2}\left(U_{t+1}-1\right)^{2}\right]\right\} \\
\Xi_{t, t+1}=\beta \frac{\lambda_{t+1}}{\lambda_{t}}
\end{gathered}
$$




$$
\begin{array}{r}
P_{t}-Q_{t}\left[1-\frac{\chi}{2}\left(\frac{I_{t}}{S_{t}^{I} I_{t-1}}-1\right)^{2}-\chi\left(\frac{I_{t}}{S_{t}^{I} I_{t-1}}\right)\left(1-\frac{I_{t}}{S_{t}^{I} I_{t-1}}\right)\right. \\
\left.=\chi E_{t}\left[\left(\frac{\Xi_{t, t+1} Q_{t+1}}{S_{t+1}^{I}}\right)\left(\frac{I_{t+1}}{I_{t}}\right)^{2}\left(\frac{I_{t+1}}{S_{t+1}^{I} I_{t}}-1\right)\right]\right]
\end{array}
$$

em que $Q_{\mathrm{t}}$, conhecido como $Q$ de Tobin, representa o multiplicador de Lagrange para a evolução do capital.

\subsection{Governo}

O governo divide-se em autoridade fiscal e monetária. O lado fiscal do governo realiza três funções: consome bens finais não duráveis e serviços, $G_{t}$; emite títulos, $B_{t+1}$; e tributa as famílias através de impostos diretos, $T_{t}$, e impostos distorcivos e indiretos, $\tau_{\mathrm{D}, \mathrm{t}}^{\mathrm{II}}$ e $\tau_{\mathrm{ND}, \mathrm{t}}^{\mathrm{II}}$. Do lado monetário, a taxa de juros, $\mathrm{R}_{\mathrm{t}}^{\mathrm{B}}$, é definida por meio de uma regra de Taylor.

\subsubsection{Política Fiscal}

O aparato fiscal incluído no modelo se baseia em Castro et al. (2011), Carvalho e Valli (2011), Cavalcanti e Vereda (2014) e Stahler e Thomas (2012). Para financiar os seus gastos, o governo possui duas fontes de recursos: por meio da gestão da dívida pública, $\mathrm{B}_{\mathrm{t}+1} /\left(\mathrm{R}_{\mathrm{t}}^{\mathrm{B}}\right)-\mathrm{B}_{\mathrm{t}}$, ou pela arrecadação de impostos, TAX $\mathrm{t}$. Do lado dos gastos, o governo consome bens finais não duráveis e serviços, enquanto que do lado das receitas, os instrumentos são $\mathrm{T}_{\mathrm{t}}, \tau_{\mathrm{D}, \mathrm{t}}^{\mathrm{II}}$ e $\tau_{\mathrm{ND}, \mathrm{t}^{\mathrm{II}}}^{\mathrm{II}}$

$$
\begin{gathered}
\frac{B_{t+1}}{R_{t}^{B}}-B_{t}=P_{N D, t} G_{t}-T A X_{t} \\
\mathrm{TAX}_{\mathrm{t}}=\tau_{\mathrm{D}, \mathrm{t}}^{\mathrm{II}} \mathrm{P}_{\mathrm{D}, \mathrm{t}} \mathrm{C}_{\mathrm{D}, \mathrm{t}}+\tau_{\mathrm{ND}, \mathrm{t}}^{\mathrm{II}} \mathrm{P}_{\mathrm{ND}, \mathrm{t}} \mathrm{C}_{\mathrm{ND}, \mathrm{t}}+\mathrm{T}_{\mathrm{t}}
\end{gathered}
$$

Todos os instrumentos seguem a mesma regra de política fiscal:

$$
\frac{Z_{t}}{Z_{s S}}=\left(\frac{Z_{t-1}}{Z_{s S}}\right)^{\gamma z}\left[\left(\frac{B_{t}}{Y_{t-1} P_{t-1}}\right)\left(\frac{Y_{s S} P_{s S}}{B_{S S}}\right)\right]^{(1-\gamma z) \phi z} S_{t}^{Z}
$$

na qual

$$
\mathrm{Z}=\left\{\mathrm{G}_{\mathrm{t}}, \tau_{\mathrm{D}, \mathrm{t}}^{\mathrm{II}}, \tau_{\mathrm{ND}, \mathrm{t}}^{\mathrm{II}}, \mathrm{T}_{\mathrm{t}}\right\}
$$


com o choque fiscal representado por

$$
\log \mathrm{S}_{\mathrm{t}}^{\mathrm{Z}}=\left(1-\rho_{\mathrm{Z}}\right) \log \mathrm{S}_{\mathrm{ss}}^{\mathrm{z}}+\rho_{\mathrm{Z}} \log \mathrm{S}_{\mathrm{t}-1}^{\mathrm{z}}+\epsilon_{\mathrm{z}, \mathrm{t}}
$$

em que $\rho^{z}$ é o componente autorregressivo e $\epsilon_{z, t} \sim N\left(0, \sigma^{z, t}\right)$.

\subsubsection{Política Monetária}

A regra de política monetária é uma regra de Taylor que estabelece que a taxa de juros nominal depende de um componente inercial e reage positivamente aos desvios da inflação e do produto em relação aos seus estados estacionários:

$$
\frac{R_{t}^{B}}{R_{S S}^{B}}=\left(\frac{R_{t-1}^{B}}{R_{S S}^{B}}\right)^{\gamma R}\left[\left(\frac{Y_{t}}{Y_{S S}}\right)^{\gamma Y}\left(\frac{\Pi_{t}}{\Pi_{S S}}\right)^{\gamma \pi}\right]^{1-\gamma R} S_{t}^{m}
$$

em que $\gamma$ R é o parâmetro de suavização da taxa de juros, $\gamma \mathrm{Y}$ e $\gamma \pi$ são os coeficientes da regra de Taylor em relação ao PIB e em relação à inflação, respectivamente, e $\mathrm{S}_{\mathrm{t}}^{\mathrm{m}}$ é o componente estocástico da regra monetária com a seguinte lei de movimento:

$$
\log S_{t}^{m}=\left(1-\rho_{m}\right) \log S_{s s}^{m}+\rho^{m} \log S_{t-1}^{m}+\epsilon_{m, t}
$$

na qual é o componente autoregressivo e , e a taxa de inflação bruta dos setores de bens duráveis e não duráveis e serviços são dadas, respectivamente, por:

$$
\Pi_{D, t}=\frac{P_{D, t}}{P_{D, t-1}}
$$

e

$$
\Pi_{N D, t}=\frac{P_{N D, t}}{P_{N D, t-1}}
$$

com

$$
\Pi_{t}=\int_{0}^{\theta N D} \Pi_{N D, j, t d j}+\int_{\theta N D}^{1} \Pi_{D, j, t d j}
$$

Assim, a taxa de inflação bruta da economia é dada por:

$$
\Pi_{t}=\gamma_{D} \Pi_{D, t}+\left(1-\gamma_{D}\right) \Pi_{N D, t}
$$




\subsection{Instituições Financeiras}

As instituições financeiras aceitam depósitos das famílias $\left(\mathrm{N}_{\mathrm{t}+1}\right)$, pagam uma taxa de remuneração por esses depósitos $\left(R_{t}^{n}\right)$ e mantêm reservas com uma proporção fixa desses depósitos $\left(\Omega_{2} N_{t+1}\right)$; emprestam na forma de capital de giro para as firmas produtoras de bens intermediários para pagamento de salários $\left(\mathrm{M}_{\mathrm{t}+1}^{\mathrm{f}}\right) \mathrm{e}$ estão sujeitas a uma taxa de inadimplência $\Omega_{1}$; emprestam também para o governo (na forma de aquisição de títulos públicos descontados por uma taxa de juros livre de risco $\left(\mathrm{B}_{\mathrm{t}+1} / \mathrm{R}_{\mathrm{t}}^{\mathrm{B}}\right)$. Assim, o problema da instituição financeira é:

$$
\max _{N_{t+1}, M_{t+1}^{f}, B_{t+1}} R_{t-1}^{f} M_{t}^{f}\left(1-\Omega_{1}\right)+B_{t}-R_{t-1}^{n} N_{t}
$$

sujeito a

$$
\frac{B_{t+1}}{R_{t}^{B}}+M_{t+1}^{f}=\left(1-\Omega_{2}\right) N_{t+1}
$$

em que $\mathrm{M}_{t+1}^{\mathrm{f}}=\mathrm{W}_{\mathrm{t}} \mathrm{L}_{\mathrm{t}}$ é a quantidade de empréstimos na forma de capital de giro para as firmas. Resolvendo-se o problema, chega-se às seguintes condições de primeira ordem:

$$
\begin{gathered}
R_{t}^{f}=\frac{R_{t}^{n}}{\left(1-\Omega_{1}\right)\left(1-\Omega_{2}\right)} \\
R_{t}^{B}=\frac{R_{t}^{n}}{\left(1-\Omega_{2}\right)}
\end{gathered}
$$

\subsection{Equilibrio}

O modelo 3 consiste na dinâmica das variáveis endógenas $\mathrm{Y}, \mathrm{Y}_{\mathrm{D}}, \mathrm{Y}_{\mathrm{ND}}, \mathrm{C}, \mathrm{C}_{\mathrm{D}}$, $\mathrm{C}_{\mathrm{ND}}, \mathrm{I}, \mathrm{G}, \mathrm{L}, \mathrm{L}_{\mathrm{D}}, \mathrm{L}_{\mathrm{ND}}, \mathrm{K}, \mathrm{K}_{\mathrm{D}}, \mathrm{K}_{\mathrm{ND}}, \mathrm{W}, \mathrm{R}, \mathrm{R}^{\mathrm{n}}, \mathrm{R}^{\mathrm{f}}, \mathrm{R}^{\mathrm{B}}, \mathrm{P}, \mathrm{P}_{\mathrm{D}}, \mathrm{P}_{\mathrm{ND}}, \Pi, \prod_{\mathrm{D}}, \prod_{\mathrm{ND}}, \tau_{\mathrm{D}}^{\mathrm{II}}, \tau_{\mathrm{ND}}^{\mathrm{II}}, \mathrm{U}, \mathrm{Q}$, $\Xi, N, M, T A X, S P, B, T, D, A, A_{D}, A_{N D}, S^{P}, S^{L}, S^{I}, S^{I I, D}, S^{I I, N D}$ e $S^{m}$, com a condição de que as condições de otimização e restrições apresentadas nas seções anteriores sejam respeitadas e os mercados apresentem-se em equilíbrio, nas seguintes formas:

a) mercado de trabalho:

$$
\mathrm{L}_{\mathrm{t}}=\mathrm{L}_{\mathrm{D}, \mathrm{t}}+\mathrm{L}_{\mathrm{ND}, \mathrm{t}}
$$

3 Vale ressaltar que uma hipótese fundamental do modelo é a de que o consumo do governo consiste apenas de bens não duráveis e serviços (equação 30). Apesar de forte, essa hipótese é razoável dado que a maior parte do consumo de bens duráveis, aqui representado pela aquisição de veículos novos, ocorre no setor privado da economia. Adicionalmente, a definição de consumo do governo (IBGE) corrobora essa simplificação, como já explicitado. 
b) mercado de capital:

$$
\mathrm{K}_{\mathrm{t}}=\mathrm{K}_{\mathrm{D}, \mathrm{t}}+\mathrm{K}_{\mathrm{ND}, \mathrm{t}}
$$

c) mercado de bens:

$$
\begin{gathered}
Y_{t}=Y_{D, t}+Y_{N D, t} \\
Y_{D, t}=C_{D, t} \\
Y_{N D, t}=C_{N D, t}+I_{t}+G_{t}
\end{gathered}
$$

\section{Propriedades do Modelo}

Nesta seção, são apresentados os procedimentos da calibragem, tratamento de dados e estimação do modelo estrutural deste trabalho.

\subsection{Calibragem}

A Tabela 2 reporta os valores dos parâmetros calibrados. $O$ fator de desconto intertemporal ( $\beta$ ), os parâmetros relacionados à regra de Taylor - isto é, o parâmetro de suavização da taxa de juros $\left(\gamma_{R}\right)$ e as sensibilidades da taxa de juros em relação ao PIB $\left(\gamma_{Y}\right)$ e à taxa de inflação $\left(\gamma_{\pi}\right)$-, o índice de rigidez dos preços $(\theta)$ e a elasticidade de substituição entre bens intermediários $(\varphi)$ foram obtidos segundo o trabalho de Castro et al. (2011). O coeficiente de aversão ao risco relativo ( $\eta$ ), a desutilidade marginal do trabalho $(\omega)$ e a taxa de depreciação do capital $(\theta)$ seguiram o estudo de Cavalcanti e Vereda (2010). Também foi calibrada a participação do capital no produto $(\alpha)$ de Kanczuk (2002). Em relação à política fiscal, supõe-se que as alíquotas de IPI sobre bens duráveis e não duráveis e serviços tem o mesmo valor no estado estacionário ( $\tau_{D, s s}^{\mathrm{II}}$ e $\tau_{\mathrm{ND}, \mathrm{IS}}^{\mathrm{II}}$ ), calibrados conforme o trabalho de Araújo e Ferreira (1999). Por fim, a taxa de inadimplência das firmas $\left(\Omega_{l}\right)$ e a taxa de compulsório $\left(\Omega_{2}\right)$ foram calibradas conforme relatório do Banco Central (BANCO CENTRAL DO BRASIL, 2014a, 2014b). 
Tabela 2 - Calibragem dos parâmetros

\begin{tabular}{|c|c|c|}
\hline Parâmetro & Valor & Fonte \\
\hline$\beta$ & 0,989 & Castro et al. (2015) \\
\hline$\eta$ & 2 & Cavalcanti e Vereda (2010) \\
\hline$\omega$ & 1,5 & Cavalcanti e Vereda (2010) \\
\hline$\zeta$ & 11 & Castro et al. (2015) \\
\hline$\alpha$ & 0,39 & Kanczuk (2002) \\
\hline$\delta$ & 0,025 & Cavalcanti e Vereda (2010) \\
\hline$\theta$ & 0,74 & Castro et al. (2015) \\
\hline$\tau_{\mathrm{D}, \mathrm{SS}}^{\mathrm{II}}$ & 0,1594 & Araújo e Ferreira (1999) \\
\hline$\tau_{\mathrm{ND}, \mathrm{SS}}{ }^{\mathrm{II}}$ & 0,1594 & Araújo e Ferreira (1999) \\
\hline$\Omega_{1}$ & 0,02 & Banco Central do Brasil (2014b) \\
\hline$\Omega_{2}$ & 0,2 & Banco Central do Brasil $\left(2014^{a}\right)$ \\
\hline$\gamma_{\mathrm{Y}}$ & 0,16 & Castro et al. (2015) \\
\hline$\gamma_{\pi}$ & 2,43 & Castro et al. (2015) \\
\hline$\gamma_{R}$ & 0,79 & Castro et al. (2015) \\
\hline$\gamma_{\mathrm{D}}$ & 0,15 & Silveira (2014) \\
\hline$\delta_{\mathrm{D}}$ & 0,05 & Depreciação contábil \\
\hline
\end{tabular}

Fonte: Elaboração própria.

\subsection{Dados}

O modelo foi estimado usando dados trimestrais de 2002T1 até 2014T4 (52 observações). Foram utilizadas as variáveis observáveis $\mathrm{R}^{\mathrm{B}}, C, G, \tau_{\mathrm{D}}^{\mathrm{II}}, \tau_{\mathrm{ND}}^{\mathrm{II}}, U, \mathrm{P}_{\mathrm{D}}$, $\mathrm{P}_{\mathrm{ND}}, L, \mathrm{Y}_{\mathrm{D}}$ e $\mathrm{Y}_{\mathrm{ND}}$, descritas na Tabela 3. 
Tabela 3 - Variáveis observáveis do modelo

\begin{tabular}{|c|c|c|}
\hline Variável & Série & Fonte \\
\hline $\mathrm{R}_{\mathrm{B}}$ & Taxa de juros selic over (\% a.m.) & Bacen/Boletim/M. Finan. \\
\hline $\mathrm{C}$ & Consumo final - famílias ( $\mathrm{R} \$$ milhões) & IBGE/SCN 2010 Trimestral \\
\hline $\mathrm{G}$ & Consumo final - adm. Pública ( $\mathrm{R} \$$ milhões) & IBGE/SCN 2010 Trimestral \\
\hline$\tau_{\mathrm{D}}{ }^{\mathrm{II}}$ & IPI - automóveis & Min. Fazenda/SRF \\
\hline$\tau_{\mathrm{ND}}{ }^{\mathrm{II}}$ & IPI - total menos automóveis & Min. Fazenda/SRF \\
\hline $\mathrm{U}$ & Utilização da capacidade instalada & $\begin{array}{l}\text { Confederação Nacional } \\
\text { da Indústria }\end{array}$ \\
\hline$P_{D}$ & $\begin{array}{l}\text { Nível de preços construído com IPCA livres } \\
\text { bens duráveis }\end{array}$ & IBGE/SNIPC \\
\hline $\mathrm{L}$ & $\begin{array}{l}\text { Número de horas trabalhadas na indústria } \\
\text { transformação }\end{array}$ & $\begin{array}{l}\text { Confederação Nacional } \\
\text { da Indústria }\end{array}$ \\
\hline$Y_{D}$ & Produção industrial - bens de cons. Duráveis & IBGE/PIM-PF \\
\hline$Y_{N D}$ & $\begin{array}{l}\text { Produção industrial - bens de cons. Não- } \\
\text { Duráveis }\end{array}$ & IBGE/PIM-PF \\
\hline
\end{tabular}

Fonte: Elaboração própria.

Como modelos DSGE são desenvolvidos para caracterizar uma economia estacionária, primeiramente deflacionamos as variáveis nominais utilizando o índice de preços ao consumidor amplo (IPCA). Em seguida, retiramos a sazonalidade e a tendência, utilizando o software X12-Arima, e aplicamos a diferença dos logaritmos, respectivamente.

\subsection{Estimação}

Dada a distribuição prior dos parâmetros, estimamos a distribuição posterior usando um processo de cadeia de Markov por meio do algoritmo Metropolis-Hastings com 100.000 interações em um valor de escala de 0,1 e 5 cadeias paralelas. A Tabela 4 e a Figura 1 apresentam as distribuições priori e posterior de cada um dos parâmetros estimados. 
Tabela 4 - Distribuição posterior do modelo

\begin{tabular}{|c|c|c|c|c|c|}
\hline Parâmetro & Prior & Posterior & $90 \%$ Intervalo & Distr. Prior & pstdev \\
\hline$\chi$ & 1 & 1,1053 & $0,78811,3950$ & gamma & 0,25 \\
\hline$\psi_{2}$ & 1 & 0,2611 & $0,21200,3085$ & gamma & 0,25 \\
\hline$\phi_{\mathrm{D}}$ & 0,925 & 0,8991 & $0,85000,9609$ & unif & 0,0433 \\
\hline$\gamma \tau_{\mathrm{D}}^{\mathrm{II}}$ & 0,5 & 0,0338 & $0,01000,0634$ & unif & 0,2829 \\
\hline$\gamma \tau_{\mathrm{ND}}^{\mathrm{II}}$ & 0,5 & 0,2262 & $0,03070,3901$ & unif & 0,2829 \\
\hline$\gamma_{G}$ & 0,5 & 0,2718 & $0,01010,5990$ & unif & 0,2829 \\
\hline$\gamma_{\mathrm{T}}$ & 0,5 & 0,2914 & $0,09630,4977$ & unif & 0,2829 \\
\hline$\varphi \tau_{\mathrm{D}}^{\mathrm{II}}$ & 0,5 & 0,2014 & $0,00030,3299$ & unif & 0,2887 \\
\hline$\varphi \tau_{\mathrm{ND}}{ }^{\mathrm{II}}$ & 0,6 & 0,2635 & $0,20000,3582$ & unif & 0,2309 \\
\hline$\varphi_{\mathrm{G}}$ & $-0,7$ & $-0,4804$ & $-0,6122-0,4000$ & unif & 0,1732 \\
\hline$\varphi_{\mathrm{T}}$ & 0,5 & 0,8802 & $0,70551,0000$ & unif & 0,2887 \\
\hline$\rho^{\mathrm{P}}$ & 0,5 & 0,488 & $0,35250,6491$ & beta & 0,1 \\
\hline$\rho^{\mathrm{L}}$ & 0,5 & 0,5365 & $0,39980,6956$ & beta & 0,1 \\
\hline$\rho^{\mathrm{m}}$ & 0,5 & 0,0518 & $0,04710,0576$ & beta & 0,1 \\
\hline$\rho^{\mathrm{A}, \mathrm{D}}$ & 0,5 & 0,2932 & $0,23030,3622$ & beta & 0,1 \\
\hline$\rho^{\mathrm{A}, \mathrm{ND}}$ & 0,5 & 0,2718 & $0,18700,3540$ & beta & 0,1 \\
\hline$\rho^{\mathrm{I}}$ & 0,5 & 0,4728 & $0,35720,6070$ & beta & 0,1 \\
\hline$\rho^{\mathrm{II}, \mathrm{D}}$ & 0,5 & 0,7449 & $0,69940,7935$ & beta & 0,1 \\
\hline$\rho^{\mathrm{II}, \mathrm{ND}}$ & 0,5 & 0,4035 & $0,30860,5096$ & beta & 0,1 \\
\hline$\rho^{\mathrm{G}}$ & 0,5 & 0,4711 & $0,29560,6408$ & beta & 0,1 \\
\hline$\rho^{\mathrm{T}}$ & 0,5 & 0,5013 & $0,39790,5949$ & beta & 0,1 \\
\hline$\varepsilon^{\mathrm{P}}$ & 1 & 0,1278 & $0,11760,1391$ & invg & Inf \\
\hline$\varepsilon^{\mathrm{L}}$ & 1 & 0,1306 & $0,11760,1441$ & invg & Inf \\
\hline$\varepsilon^{\mathrm{m}}$ & 1 & 0,1614 & $0,13060,1915$ & invg & Inf \\
\hline$\varepsilon^{\mathrm{A}, \mathrm{D}}$ & 1 & 0,2151 & $0,1782 \quad 0,2545$ & invg & Inf \\
\hline$\varepsilon^{\mathrm{A}, \mathrm{ND}}$ & 1 & 0,2355 & $0,18420,2815$ & invg & Inf \\
\hline$\varepsilon^{\mathrm{I}}$ & 1 & 0,2587 & $0,20590,3135$ & invg & Inf \\
\hline
\end{tabular}




\begin{tabular}{l|r|r|l|l|l}
\multicolumn{2}{l}{ conclusão. } \\
\hline Parâmetro & Prior & Posterior & $\mathbf{9 0 \% \text { Intervalo }}$ & Distr. Prior & pstdev \\
\hline$\varepsilon^{\mathrm{II}, \mathrm{D}}$ & 1 & 0,2769 & $0,23060,3190$ & invg & Inf \\
\hline$\varepsilon^{\mathrm{II}, \mathrm{ND}}$ & 1 & 0,1281 & $0,11760,1393$ & invg & Inf \\
\hline$\varepsilon^{\mathrm{G}}$ & 1 & 0,126 & $0,11760,1352$ & invg & Inf \\
\hline$\varepsilon^{\mathrm{T}}$ & 1 & 0,2692 & $0,19850,3447$ & invg & Inf \\
\hline
\end{tabular}

Fonte: Elaboração própria.

Figura 1 - Prior e posterior do modelo
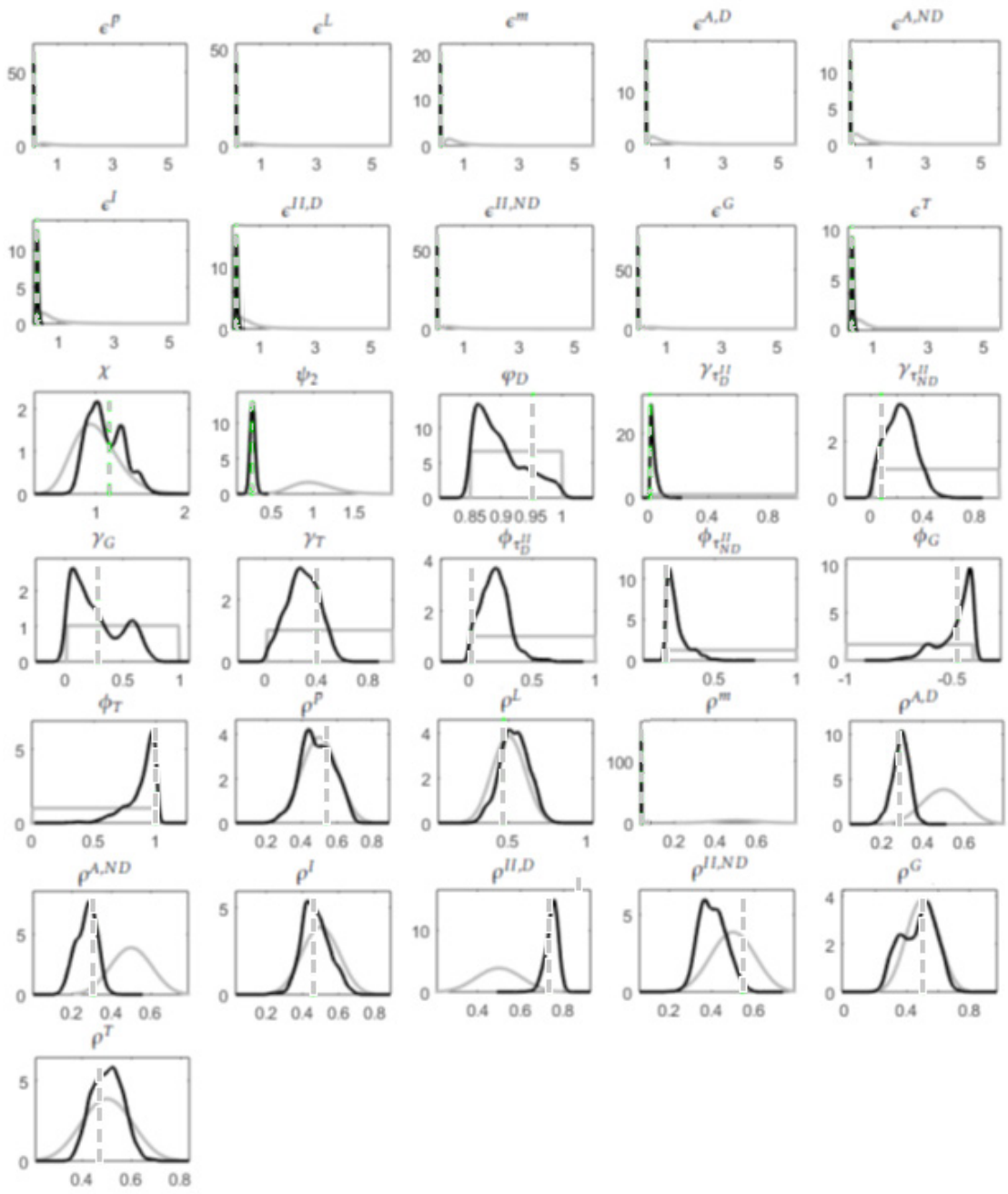

Fonte: Elaboração própria. 


\section{Resultados}

Nesta seção, analisamos o comportamento das principais variáveis macroeconômicas diante de choques exógenos e não antecipados de política fiscal em relação ao consumo de bens duráveis e não duráveis. O modelo apresenta funções impulso-resposta bem-comportadas e consistentes com a evidência empírica. ${ }^{4}$

Iniciamos essa análise com a redução na alíquota do imposto indireto sobre bens duráveis na magnitude de um desvio padrão $(0,2769)$, exposta na Figura 2.

Figura 2 - Resposta ao choque na alíquota do imposto indireto sobre bens duráveis
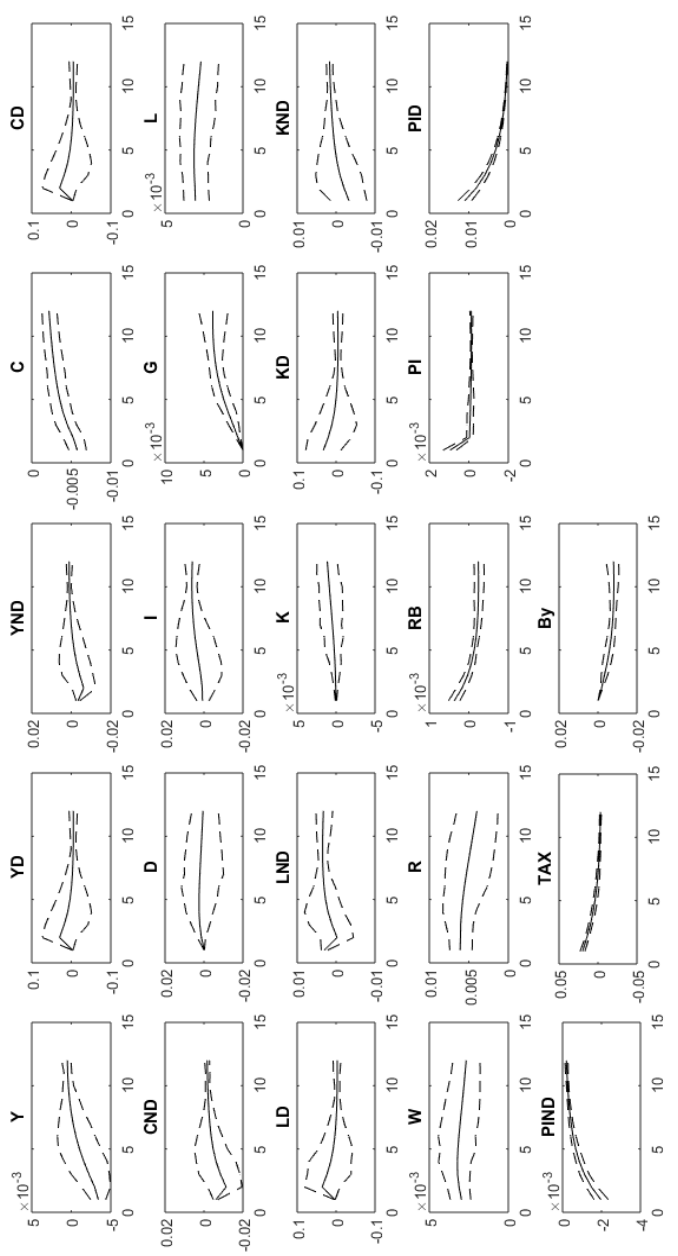

Fonte: Elaboração própria.

4 O Apêndice B apresenta as funções impulso-resposta para os outros choques do modelo. 
Esse choque aumenta a demanda por bens duráveis, e a oferta desse bem responde positivamente, bem como seus insumos $\left(K_{D}, L_{D}\right)$. Por a desoneração desse imposto não ter sido suficiente para aumentar a renda disponível, as famílias ajustam o seu orçamento para compensar o aumento no consumo de bens duráveis, e, dessa forma, a demanda por bens não duráveis diminui. No agregado, nota-se que a queda na demanda por bens não duráveis mais que compensou o aumento na demanda por bens duráveis, resultando na queda do produto agregado. Do lado fiscal, há uma melhora na arrecadação total, aumento no gasto corrente e queda no nível da dívida pública em proporção do PIB. Esses efeitos, em média, possuem duração de 10 trimestres.

Supondo que o choque de política fiscal tivesse sido uma redução da alíquota de impostos indiretos para o setor de bens não duráveis $(0,1281)$, teríamos resultados distintos do anterior, conforme a Figura 3. Dada a relevância desse bem na cesta de consumo das famílias, ${ }^{5}$ essa desoneração melhora a renda disponível das famílias, e, dessa forma, esse agente aumenta o seu consumo agregado (consumo de bens duráveis e não duráveis), fato que torna o produto agregado positivo. Do lado fiscal, da mesma forma que na desoneração anterior, ocorre aumento na arrecadação total, aumento no gasto corrente e melhora na dívida pública em proporção do PIB. O efeito dessa desoneração durou cinco trimestres.

5 A produção de bens não duráveis é de cerca de $85 \%$ da produção total da economia brasileira (SILVEIRA, 2014). 
Figura 3 - Resposta ao choque na alíquota do imposto indireto sobre bens não duráveis e serviços
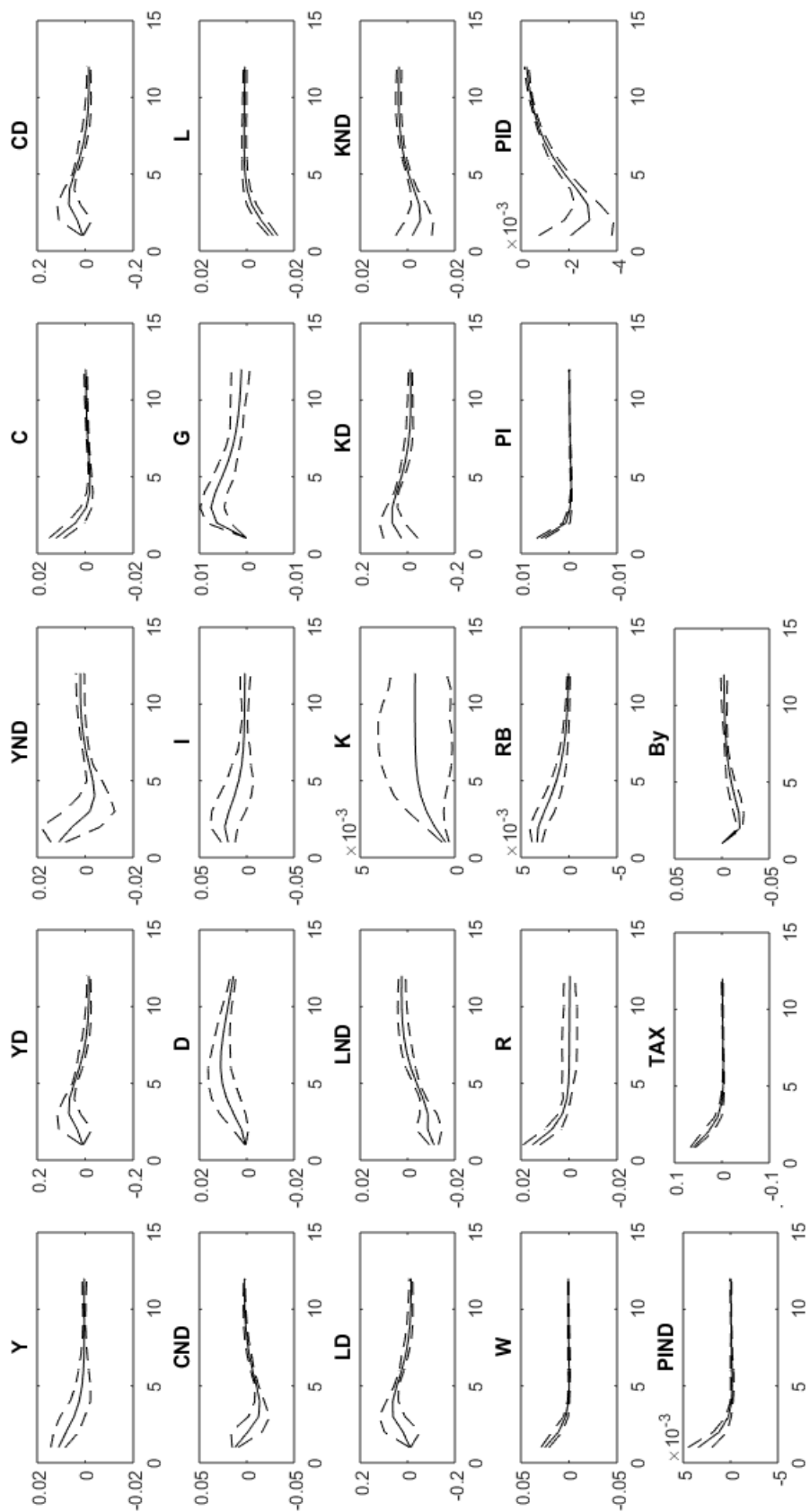

Fonte: Elaboração própria. 
Outra forma de avaliar o desempenho das políticas de desoneração do IPI é por meio da decomposição dos choques para o PIB para o período estudado, conforme a Figura 4.

Figura 4 - Decomposição dos choques para o PIB

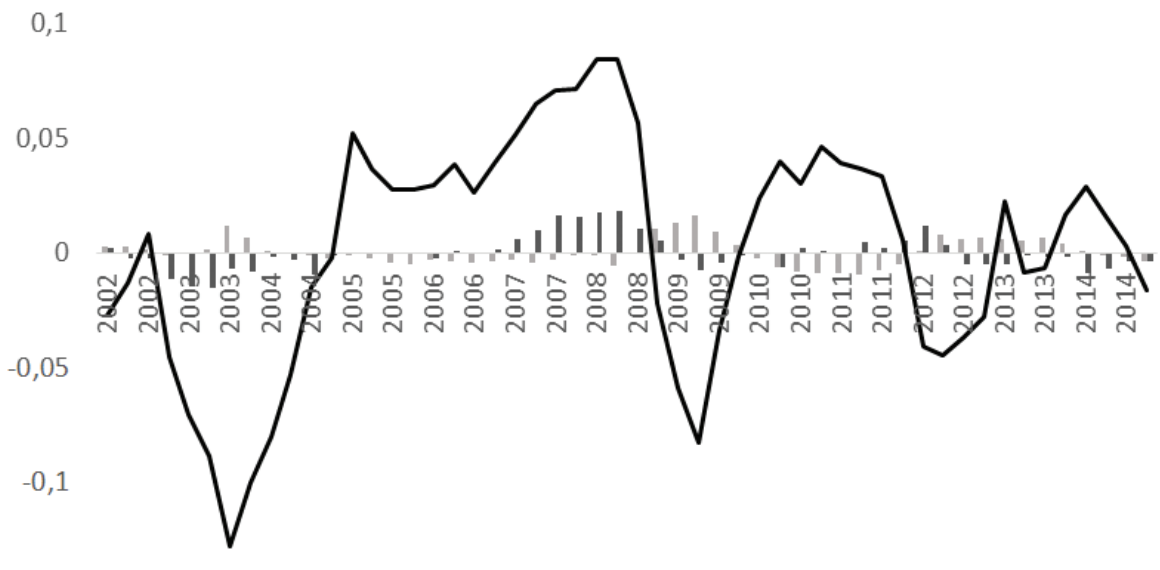

$-0,15$

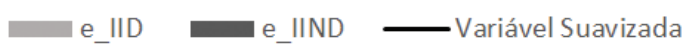

Fonte: Elaboração própria.

O choque objeto deste trabalho - desoneração do imposto indireto sobre o consumo de bens duráveis - teve pouca relevância para o desempenho do PIB por todo o período estudado. As desonerações pós-crise de 2008 sobre o IPI para bens duráveis contribuíram, aproximadamente, com 1,5\% para a recuperação da crise, mas, em seguida, colaboraram negativamente no período de 2010 até o final de 2011 . Outro período positivo teve início em 2012 e durou nove trimestres, mas com uma amplitude ainda menor do que a anterior.

\section{Considerações Finais}

A eclosão da crise financeira internacional de 2008 colocou o uso da política fiscal novamente no radar dos formuladores de políticas públicas como forma de impulsionar a atividade econômica.

Devido à enorme incerteza na literatura a respeito dos efeitos da política fiscal expansionista sobre o nível de atividade, no presente trabalho analisamos a desoneração do IPI sobre automóveis com o objetivo de fornecer contribuições em duas direções: a) estimar e calibrar um modelo DSGE de pequena escala, para 
o Brasil, que incorpore esse aspecto; e b) analisar o impacto específico dessa política nas principais variáveis macroeconômicas à luz do modelo.

A inovação dessa abordagem se deve à exploração de uma economia de dois setores, a saber: bens duráveis e bens não duráveis e serviços. Uma característica importante dessa economia é que os agentes não somente têm que escolher entre consumo e lazer no tempo, mas também o tipo de bem (durável ou não durável e serviços) que será consumido.

Os resultados do modelo proposto sugerem que a redução da alíquota do IPI sobre bens duráveis não foi a política mais adequada para estimular a atividade econômica. Com o impacto da desoneração desse imposto, o produto agregado recua para um valor abaixo do seu estado estacionário. Supondo-se que o choque de política fiscal tivesse sido uma redução da alíquota do imposto indireto para toda a economia, ou para o setor de bens não duráveis e serviços (que detém uma participação maior na economia), o modelo proposto sugere que essa política teria sido mais adequada para reanimar a atividade econômica.

Dessa maneira, os resultados da nossa análise podem ser úteis para avaliar a viabilidade de outros programas de estímulo no setor de bens duráveis no futuro. É necessário, assim, que as autoridades avaliem cuidadosamente os custos e benefícios de políticas implementadas, sob pena de comprometerem o equilíbrio orçamentário e a sustentabilidade da dívida pública.

Por fim, os resultados sugerem estudos adicionais, além do mais com a inclusão de indivíduos não ricardianos e regras de política fiscal alternativas no modelo.

\section{Referências}

ADDA, J.; COOPER, R. Balladurette and Juppette: a discrete analysis of scrapping subsidies. Journal of political Economy, v. 108, n. 4, p. 778-806, 2000.

BANCO CENTRAL DO BRASIL. Estatísticas monetárias e de crédito. Disponível em: <https://www.bcb.gov.br/htms/notecon2-p.asp >. Acesso em: 7 mar. 2014b.

. Quadro Resumo - BCB. Disponível em:<https://www.bcb.gov.br/htms/ novaPaginaSPB/Resumo_das_normas_dos_compuls\%C3\%B3rios.pdf $>$. Acesso em: 2 mar. 2014a.

BARRO, R. J. Are government bonds net wealth? Journal of Political Economy, v. 82, n. 6, p. 1095-1117, 1974.

. On the determination of the public debt. Journal of political Economy, v. 87, n. 5, part. 1, p. 940-971, 1979.

BLANCHARD, O.; PEROTTI, R. An empirical characterization of the dynamic effects of changes in government spending and taxes on output. The Quarterly Journal of Economics, v. 117, n. 4, p. 1329-1368, 2002. 
CARVALHO, F. A.; VALLI, M. Fiscal policy in Brazil through the Lens of an Estimated DSGE Model. Brasília, DF: Banco Central do Brasil, Apr. 2011. (Working Paper Series, n. 240).

CASTRO, M. R. et al. SAMBA: Stochastic analytical model with a bayesian approach. Brazilian Review of Econometrics, v. 35, n. 2, p. 103-170, 2015.

CAVALCANTI, M. A. F. H. Política fiscal e nível de atividade: breve resenha da literatura empírica e lições para o Brasil. Revista Economia 8 Tecnologia, v. 5, n. 4, p. 15-23, 2009.

; VEREDA, L. Multiplicadores dos gastos públicos em um modelo DSGE para o Brasil. Rio de Janeiro: Ipea, 2014. (Nota Técnica).

Propriedades dinâmicas de um modelo DSGE com parametrizações alternativas para o Brasil. Rio de Janeiro: Ipea, 2011. (Texto para Discussão, n. 1588).

COGAN, J. F. et al. New Keynesian versus old Keynesian government spending multipliers. Journal of Economic dynamics and control, v. 34, n. 3, p. 281-295, 2010.

CONJUR. Carros ficam mais três meses livres do IPI. Disponível em: < https://www.conjur. com.br/2009-jun-29/governo-prorroga-tres-isencao-ipi-carros>. Acesso em: 12 fev. 2014.

FERREIRA, P. C. G.; ARAÚJO, C. H. V. Reforma tributária, efeitos alocativos e impactos de bem-estar. Revista Brasileira de Economia, v. 53, n. 2, p. 133-166, 1999.

FORNI, L.; MONTEFORTE, L.; SESSA, L. The general equilibrium effects of fiscal policy: estimates for the euro area. Journal of Public Economics, v. 93, n. 3-4, p. 559-585, 2009.

INTERNATIONAL MONETARY FUND. Fiscal monitor: public expenditure reform: making difficult choices. Washington, DC: IMF, 2014. Disponível em: < http://www.imf.org/external/ pubs/ft/fm/2014/01/fmindex.htm>. Acesso em: 6 jun. 2014.

KANCZUK, F. Juros reais e ciclos reais brasileiros. Revista Brasileira de Economia, v. 56, n. 2, p. 249-267, 2002.

LEACH, J. A course in public economics. Cambridge, MA: Cambridge University Press, 2004.

MENDONÇA, M. J.; MEDRANO, L. A.; SACHISDA, A. Avaliando os efeitos da política fiscal no Brasil: resultados de um procedimento de identificação agnóstica. Rio de Janeiro: Ipea, 2009. (Texto para Discussão, n. 1377).

MIAN, A.; SUFI, A. The effects of fiscal stimulus: Evidence from the 2009 cash for clunkers program. The Quarterly Journal of Economics, v. 127, n. 3, p. 1107-1142, 2012.

PERES, M. A. Os efeitos dinâmicos da política fiscal sobre a atividade econômica: um estudo para o caso brasileiro. Dissertação (Mestrado em Economia) - Departamento de Economia, Universidade de Brasília, Brasília, DF, 2006.

PEROTTI, R.; REIS, R.; RAMEY, V. In search of the transmission mechanism of fiscal policy [with comments and discussion]. NBER Macroeconomics Annual, v. 22, p. 169-249, 2007. 
PIRES, M. C. C. Multiplicadores fiscais no Brasil: uma contribuição ao debate sobre políticas fiscais anticíclicas. In: ENCONTRO NACIONAL DE ECONOMIA DA ANPEC, 37., 2009, Foz do Iguaçu. Anais... Foz do Iguaçu: Anpec, 2009.

RECEITA FEDERAL DO BRASIL. IPI (Imposto sobre produtos industrializados) - isenção para taxistas, pessoas com deficiência e outros. Disponível em: $<$ http://idg.receita.fazenda. gov.br/acesso-rapido/legislacao/legislacao-por-assunto/isencao-do-ipi>. Acesso em: 17 jun. 2015.

SAHA, D.; VON WEIZSÄCKER, J. EU stimulus packages. Estimating the size of the European stimulus packages for 2009: an update. Bruegel Policy Contribution, 2009.

SILVEIRA, M. A. C. Gastos com bens duráveis no ciclo da vida das famílias brasileiras. Brasília, DF: Ipea, 2014. (Texto para Discussão, n. 1984).

SMETS, F.; WOUTERS, R. An estimated dynamic stochastic general equilibrium model of the euro area. Journal of the European Economic Association, v. 1, n. 5, p. 1123-1175, 2003.

STÄHLER, N.; THOMAS, C. FiMod-A DSGE model for fiscal policy simulations. Economic Modelling, v. 29, n. 2, p. 239-261, 2012.

TELES, V. K.; JÚNIOR, C. J. C.; ROSA, R. M. Investment-specific technological change and the Brazilian macroeconomy. Brazilian Review of Econometrics, v. 35, n. 2, p. 233-264, 2015.

UNIVERSITY OF TORONTO. Munk Centre for International Studies. G20 Research Group. Declaration of the summit on financial markets and the world economy. Toronto, Canada, 2008. Disponível em: < http://www.g20.utoronto.ca/2008/2008declaration1115.html > . Acesso em: 15 abr. 2014.

WHELAN, K. A two-sector approach to modeling US NIPA data. Journal of Money, Credit and Banking, v. 35, n. 4, p. 627-656, 2003.

\section{Apêndice A - Equações log-linear do modelo}

Da equação 2:

$$
\tilde{C}_{t}=\gamma_{D} \widetilde{D}_{t+1}+\left(1-\gamma_{D}\right) \tilde{C}_{N D, t}
$$

Da equação 3:

$$
\widetilde{D}_{t+1}=\delta_{D} \tilde{C}_{D, t}+\left(1-\delta_{D}\right) \widetilde{D}_{t}
$$

Da equação 4:

$$
\tilde{S}_{t}^{P}=\rho^{P} \tilde{S}_{t-1}^{P}+\epsilon_{t}^{P}
$$


Da equação 5:

$$
\tilde{S}_{t}^{L}=\rho^{L} \tilde{S}_{t-1}^{L}+\epsilon_{t}^{L}
$$

Da equação 6,

$$
\begin{aligned}
C_{D, S S} P_{D, s s}\left[\left(\tilde{C}_{D, t}\right.\right. & \left.\left.+\tilde{P}_{D, t}\right)\left(1+\tau_{D, s s}^{I I}\right)+\tau_{D, s s}^{I I} \tilde{\tau}_{D, s s}^{I I}\right] \\
& +C_{N D, S S} P_{N D, s s}\left[\left(\tilde{C}_{N D, t}+\tilde{P}_{N D, t}\right)\left(1+\tau_{N D, s s}^{I I}\right)+\tau_{N D, s s}^{I I} \tilde{\tau}_{N D, s s}^{I I}\right]+N_{s s} \widetilde{N}_{t+1} \\
& =W_{s s} L_{s s}\left(\widetilde{W}_{t}+\tilde{L}_{t}\right)+R_{s s}^{n} N_{S s}\left(\tilde{R}_{t}^{n}+\widetilde{N}_{t}\right)-T_{s S} \widetilde{T}_{t}
\end{aligned}
$$

Da equação 7:

$$
\begin{gathered}
\left(\frac{\gamma_{D}}{1-\gamma_{D}}\right)^{\eta} P_{D, s s} D_{s s}\left(\frac{1-\varphi_{D}}{\varphi_{D}}\right)^{\eta}\left\{\left[\tilde{P}_{D, t}+\left(\frac{1-\varphi_{D}}{\varphi_{D}}\right) \eta \widetilde{D}_{t+1}\right]\left(1+\tau_{D, s s}^{I I}\right)+\tau_{D, s s}^{I I} \tilde{\tau}_{D, t}^{I I}\right\} \\
=P_{N D, s s} C_{N D, s s}^{\left(\frac{1-\varphi_{D}}{\varphi_{D}}\right) \eta}\left\{\left[\tilde{P}_{N D, t}+\left(\frac{1-\varphi_{D}}{\varphi_{D}}\right) \eta \tilde{C}_{N D, t}\right]\left(1+\tau_{N D, s s}^{I I}\right)\right. \\
\left.+\tau_{N D, s s}^{I I} \tilde{\tau}_{N D, t}^{I I}\right\}
\end{gathered}
$$

Da equação 8:

$$
\begin{gathered}
\left(1-\gamma_{D}\right)^{\eta} P_{N D, s S} C_{N D, s s}^{\left(\frac{1-\varphi_{D}}{\varphi_{D}}\right) \eta} L_{S S}^{\omega}\left\{\left[\tilde{S}_{t}^{L}+\tilde{P}_{N D, t}+\left(\frac{1-\varphi_{D}}{\varphi_{D}}\right) \eta \tilde{C}_{N D, t}+\omega \tilde{L}_{t}\right]\left(1+\tau_{N D, s s}^{I I}\right)\right. \\
\left.+\tau_{N D, s s}^{I I} \tilde{T}_{N D, t}^{I I}\right\}=W_{S S} C_{s s}^{\left(1-\varphi_{D}\right) \eta}\left[\widetilde{W}_{t}+\left(1-\varphi_{D}\right) \eta \tilde{C}_{t}\right]
\end{gathered}
$$

Da equação 9:

$$
\tilde{R}_{t+1}^{n}=-\Xi_{t, t+1}
$$

Da equação 13:

$$
\begin{gathered}
\tilde{Y}_{D, t}=\tilde{A}_{D, t}+\alpha \widetilde{K}_{D, t}+(1-\alpha) \tilde{L}_{D, t} \\
\tilde{Y}_{N D, t}=\tilde{A}_{N D, t}+\alpha \widetilde{K}_{N D, t}+(1-\alpha) \tilde{L}_{N D, t}
\end{gathered}
$$

Da equação 14:

$$
\tilde{A}_{D, t}=\rho^{A, D} \tilde{A}_{D, t-1}+\epsilon_{t}^{A, D}
$$


Da equação 15:

$$
\tilde{A}_{N D, t}=\rho^{A, N D} \tilde{A}_{N D, t-1}+\epsilon_{t}^{A, N D}
$$

Da combinação das equações 17 e 18:

$$
\begin{gathered}
\tilde{R}_{t}^{f}+\widetilde{W}_{t}-\tilde{R}_{t}=\widetilde{K}_{D, t}-\tilde{L}_{D, t} \\
\tilde{R}_{t}^{f}+\widetilde{W}_{t}-\tilde{R}_{t}=\widetilde{K}_{N D, t}-\tilde{L}_{N D, t}
\end{gathered}
$$

Da equação 19:

$$
\begin{gathered}
C M_{D, t}=(1-\alpha)\left(\tilde{R}_{t}^{f}+\widetilde{W}_{t}\right)+\alpha \tilde{R}_{t}-\tilde{A}_{D, t} \\
C M_{N D, t}=(1-\alpha)\left(\tilde{R}_{t}^{f}+\widetilde{W}_{t}\right)+\alpha \tilde{R}_{t}-\tilde{A}_{N D, t}
\end{gathered}
$$

Da combinação das equações 20 e 21:

$$
\begin{gathered}
\tilde{\pi}_{D, t}=\beta \tilde{\pi}_{D, t+1}+\left[\frac{(1-\theta)(1-\beta \theta)}{\theta}\right]\left(C M_{D, t}-\tilde{P}_{D, t}\right) \\
\tilde{\pi}_{N D, t}=\beta \tilde{\pi}_{N D, t+1}+\left[\frac{(1-\theta)(1-\beta \theta)}{\theta}\right]\left(C M_{N D, t}-\tilde{P}_{N D, t}\right)
\end{gathered}
$$

Da equação 23:

$$
\widetilde{K}_{t+1}=(1-\delta) \widetilde{K}_{t}+\delta \tilde{I}_{t}
$$

Da equação 24:

$$
\tilde{S}_{t}^{I}=\rho^{I} \tilde{S}_{t-1}^{I}+\epsilon_{t}^{I}
$$

Da equação 25:

$$
\frac{R_{S S}}{P_{S S}}\left(\tilde{R}_{t}-\tilde{P}_{t}\right)=\psi_{2} U_{t}
$$

Da equação 26:

$$
\frac{Q_{s s}}{\Xi_{s S}}\left(\tilde{Q}_{t}-\widetilde{\Xi}_{t, t+1}\right)=(1-\delta) Q_{s s} \tilde{Q}_{t+1}-R_{s s}\left(\tilde{R}_{t+1}+\widetilde{U}_{t+1}\right)+\psi_{1} \widetilde{U}_{t+1}
$$


Da equação 27:

$$
\begin{gathered}
\widetilde{\Xi}_{t, t+1}=\left[\tilde{S}_{t+1}^{P}-\tilde{S}_{t}^{P}+\eta\left(\frac{1-\varphi_{D}}{\varphi_{D}}\right)\left(\tilde{C}_{N D, t}-\tilde{C}_{N D, t+1}\right)+\eta\left(1-\varphi_{D}\right)\left(\tilde{C}_{t+1}-\tilde{C}_{t}\right)-\tilde{\pi}_{t+1}\right] \\
+\left[\frac{\tau_{N D, s s}^{I I}}{1+\tau_{N D, s s}^{I I}}\right]\left(\tilde{\tau}_{N D, t}^{I I}-\tilde{\tau}_{N D, t+1}^{I I}\right)
\end{gathered}
$$

Da equação 28:

$$
P_{s s} \tilde{P}_{t}+Q_{s s} \tilde{Q}_{t}+\chi Q_{s s}\left[\tilde{S}_{t}^{I}-\left(\tilde{I}_{t}-\tilde{I}_{t-1}\right)\right]=\chi Q_{s s} \Xi_{s s}\left[\tilde{S}_{t+1}^{I}-\left(\tilde{I}_{t+1}-\tilde{I}_{t}\right)\right]
$$

Da equação 29:

$$
\frac{B_{S S}}{R_{S S}^{B}}\left(\tilde{B}_{t+1}-\tilde{R}_{t}^{B}\right)-B_{S S} \tilde{B}_{t}=P_{N D, s S} G_{s S}\left(\tilde{P}_{N D, t}+\tilde{G}_{t}\right)-T A X_{S S} \widetilde{T A X}_{t}
$$

Da equação 30:

$$
\begin{aligned}
T A X_{S S} \widetilde{T A X}_{t}= & \tau_{D, s s}^{I I} P_{D, s s} C_{D, s s}\left(\tilde{\tau}_{D, t}^{I I}+\tilde{P}_{D, t}+\tilde{C}_{D, t}\right)+\tau_{N D, s S}^{I I} P_{N D, s S} C_{N D, s s}\left(\tilde{\tau}_{N D, t}^{I I}+\tilde{P}_{N D, t}+\tilde{C}_{N D, t}\right) \\
& +T_{s S} \widetilde{T}_{t}
\end{aligned}
$$

Da equação 32:

$$
\tilde{S}_{t}^{Z}=\rho^{Z} \tilde{S}_{t-1}^{Z}+\epsilon_{t}^{Z}
$$

Da definição do estoque da dívida pública em proporção do PIB:

$$
\tilde{B}_{Y, t}=\tilde{B}_{t}-\tilde{Y}_{t-1}-\tilde{P}_{t-1}
$$

Da equação 39:

$$
\begin{gathered}
\frac{B_{S S}}{R_{S S}^{B}}\left(\tilde{B}_{t+1}-\tilde{R}_{t}^{B}\right)+M_{S S}^{f} \widetilde{M}_{t+1}^{f}=\left(1-\Omega_{2}\right) N_{S S} \widetilde{N}_{t+1} \\
\tilde{M}_{t+1}^{f}=\widetilde{W}_{t}+\tilde{L}_{t}
\end{gathered}
$$

Da equação 40:

$$
\tilde{R}_{t}^{B}=\tilde{R}_{t}^{n}
$$

Da equação 41:

$$
\tilde{R}_{t}^{f}=\tilde{R}_{t}^{n}
$$


Da equação 33:

$$
\tilde{R}_{t}^{B}=\gamma_{R} \tilde{R}_{t}^{B}+\left(1-\gamma_{R}\right)\left(\gamma_{Y} \tilde{Y}_{t}+\gamma_{\pi} \tilde{\pi}\right)+\tilde{S}_{t}^{m}
$$

Da equação 34:

$$
\tilde{S}_{t}^{m}=\rho^{m} \tilde{S}_{t-1}^{m}+\epsilon_{t}^{m}
$$

Da equação 35:

$$
\tilde{\pi}_{D, t}=\tilde{P}_{D, t}-\tilde{P}_{D, t-1}
$$

Da equação 36:

$$
\tilde{\pi}_{N D, t}=\tilde{P}_{N D, t}-\tilde{P}_{N D, t-1}
$$

Da equação 37:

$$
\tilde{\pi}_{t}=\tilde{P}_{t}-\tilde{P}_{t-1}
$$

Da equação 44:

$$
Y_{S S} \tilde{Y}_{t}=Y_{D, S S} \tilde{Y}_{D, t}+Y_{N D, S S} \tilde{Y}_{N D, t}
$$

Da equação 45:

$$
\tilde{Y}_{D, t}=\tilde{C}_{D, t}
$$

Da equação 46:

$$
Y_{N D, s S} \tilde{Y}_{N D, t}=C_{N D, S S} \tilde{C}_{N D, t}+I_{S S} \tilde{I}_{t}+G_{S S} \tilde{G}_{t}
$$

Da equação 42:

$$
L_{s S} \tilde{L}_{t}=L_{D, s s} \tilde{L}_{D, t}+L_{N D, s s} \tilde{L}_{N D, t}
$$

Da equação 43:

$$
K_{S S} \widetilde{K}_{t}=K_{D, s S} \widetilde{K}_{D, t}+K_{N D, s S} \widetilde{K}_{N D, t}
$$




\section{Apêndice B - IRFs de outros choques do modelo}

Figura 5 - Resposta ao choque monetário
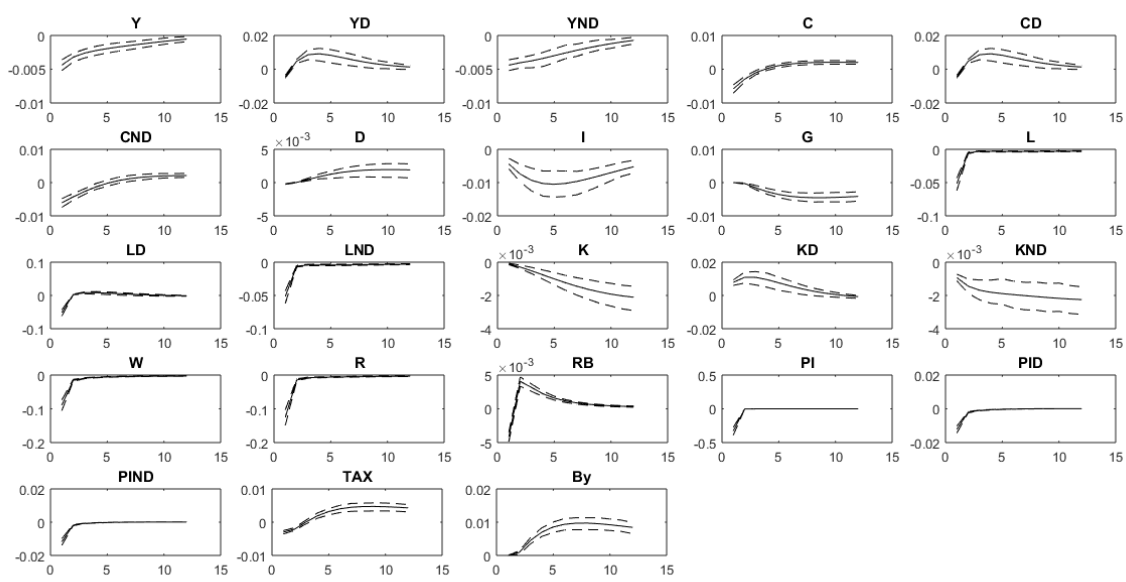

Fonte: Elaboração própria.

Figura 6 - Resposta ao choque de produtividade no setor de bens duráveis
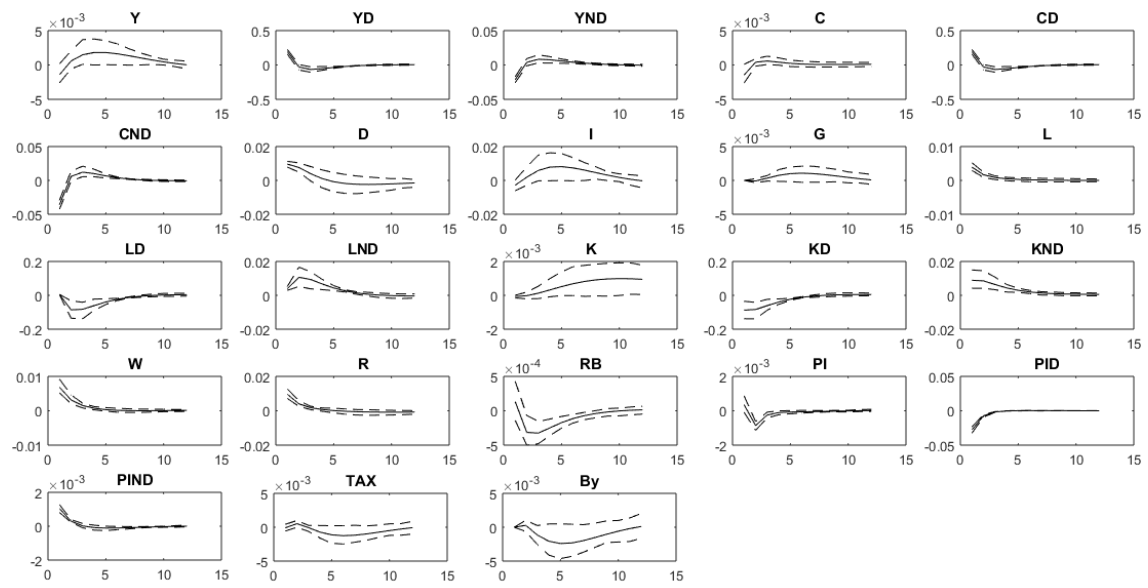

Fonte: Elaboração própria. 
Figura 7 - Resposta ao choque de produtividade no setor de bens não duráveis
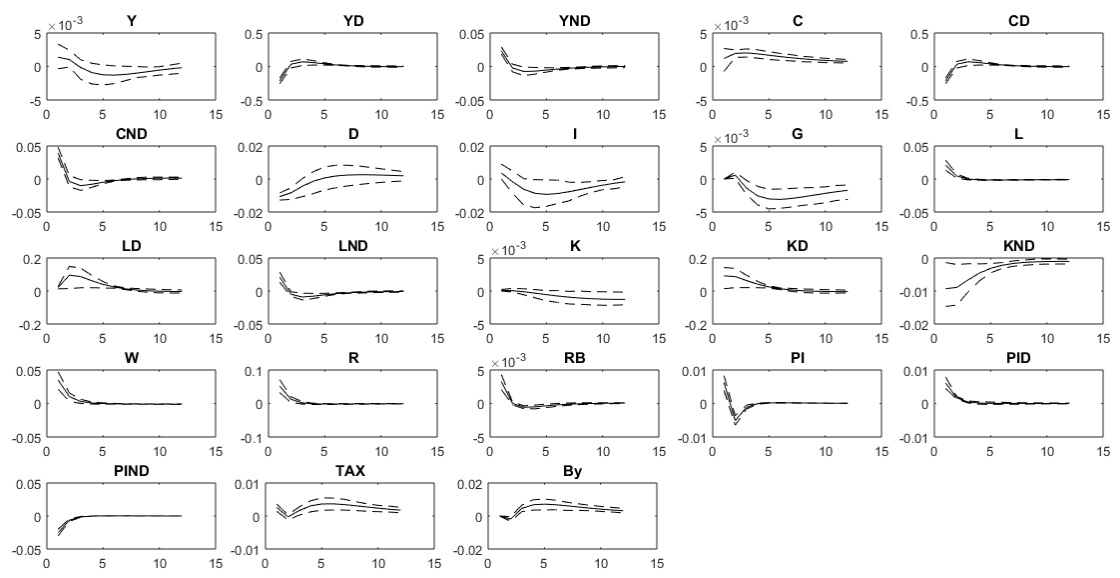

Fonte: Elaboração própria.

Figura 8 - Resposta ao choque de produtividade do investimento
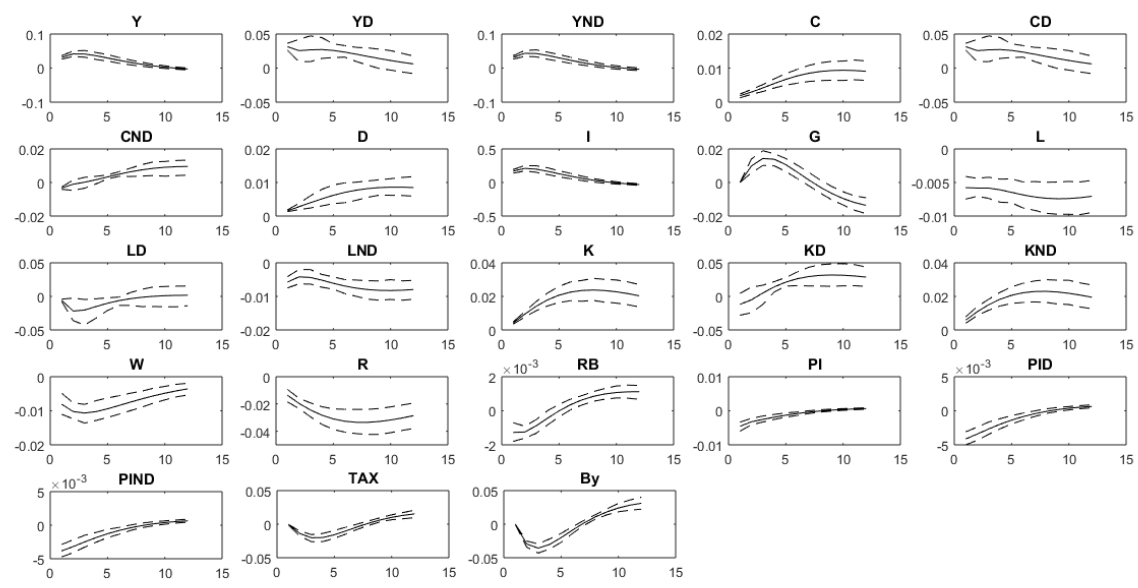

Fonte: Elaboração própria. 
Figura 9 - Resposta ao choque de preferências
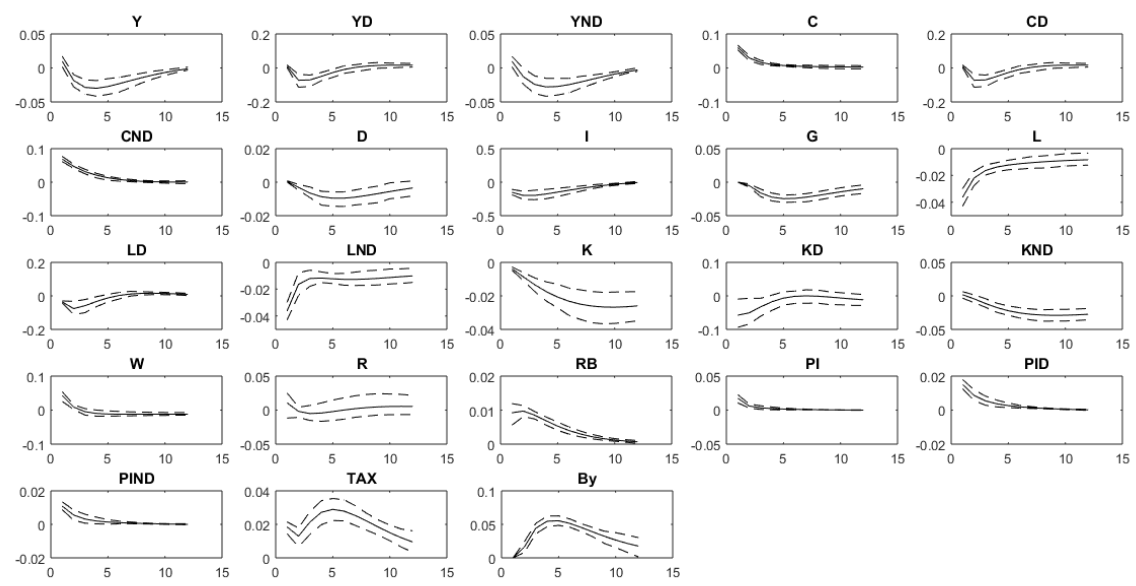

Fonte: Elaboração própria.

Figura 10 - Resposta ao choque de oferta de trabalho
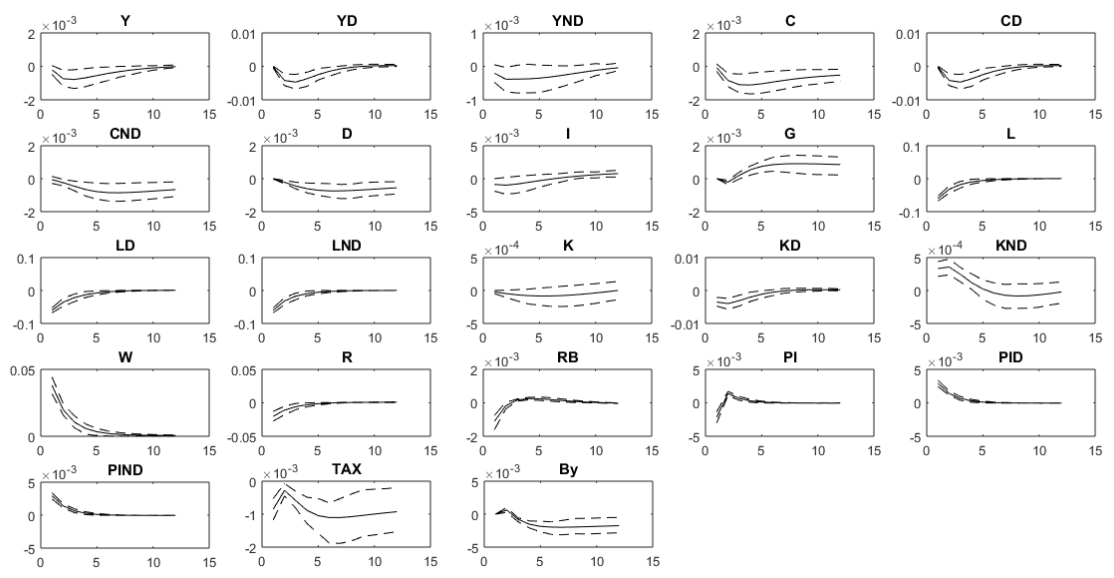

Fonte: Elaboração própria. 
Figura 11 - Resposta ao choque de gasto do governo
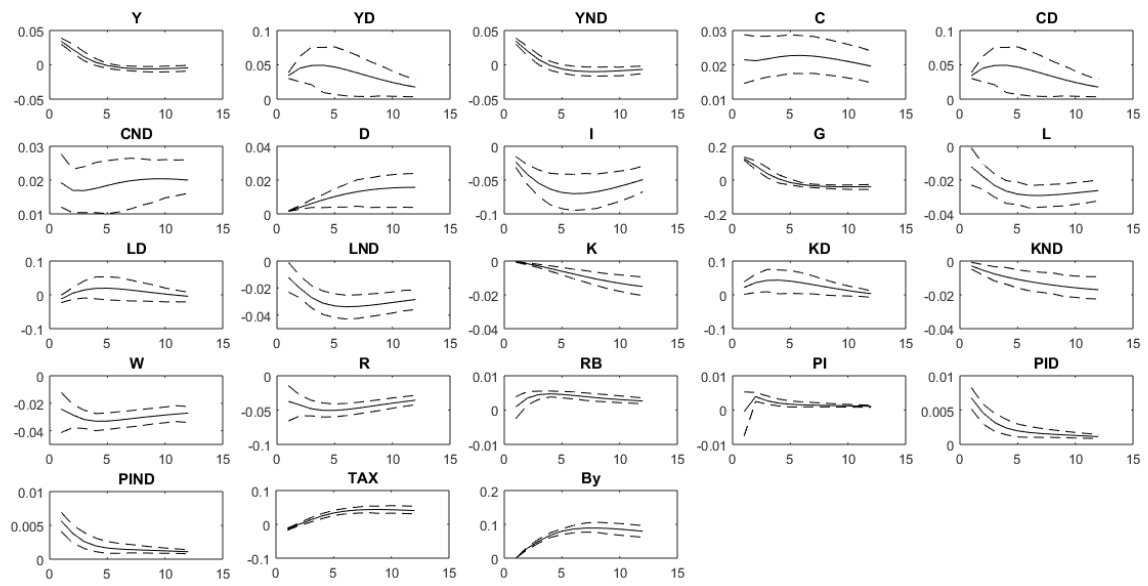

Fonte: Elaboração própria.

Figura 12 - Resposta ao choque de imposto lump-sum
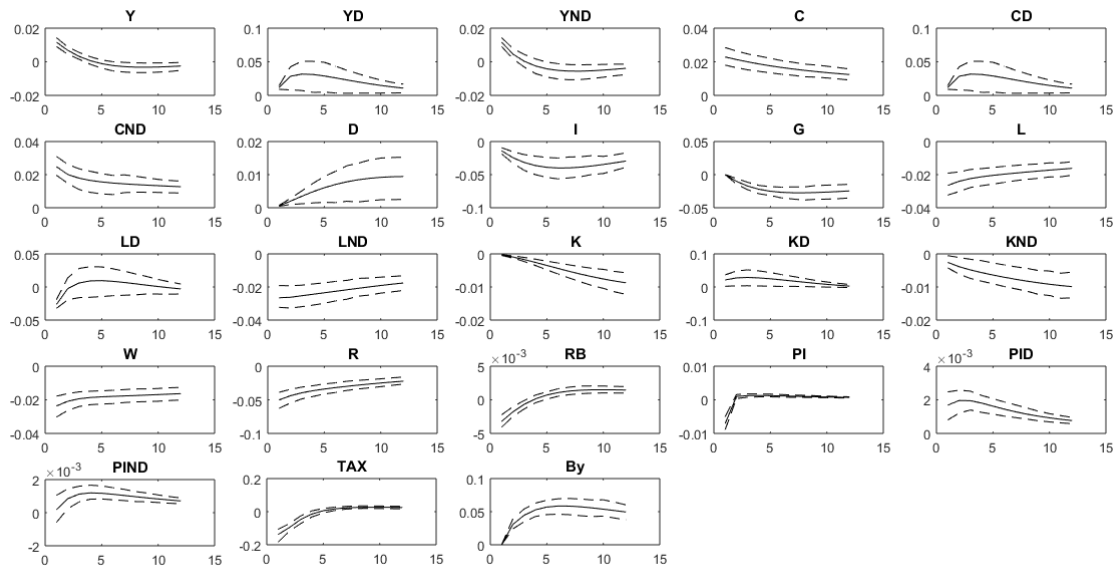

Fonte: Elaboração própria.

Recebido em: 29/09/2016.

Aceito em: 24/02/2017. 